\title{
MIXED NORM ESTIMATES FOR CERTAIN MEANS
}

\author{
LENNART BÖRJESON
}

ABSTRACT. We obtain estimates of the mean

$$
F_{x}^{\gamma}(t)=C_{\gamma} \int_{|y|<1}\left(1-|y|^{2}\right)^{\gamma} f(x-t y) d y
$$

in mixed Lebesgue and Sobolev spaces. They generalize earlier estimates of the spherical mean $F_{x}^{-1}(t)=C \int_{S^{n-1}} f(x-t y) d S(y)$ and of solutions of the wave equation $\Delta_{x} u=\partial^{2} u / \partial t^{2}$.

Introduction. For $f \in C_{0}^{\infty}\left(\mathbf{R}^{n}\right)$ and $\gamma>-1$ we define the mean

$$
F_{x}^{\gamma}(t)=\frac{2^{-\gamma}(2 \pi)^{-\frac{n}{2}}}{\Gamma(1+\gamma)} \int_{|y|<1}\left(1-|y|^{2}\right)^{\gamma} f(x-t y) d y,
$$

$x \in \mathbf{R}^{n}, t \in \mathbf{R}$. $\Gamma$ is the gamma function. A computation of the Fourier transform of $F^{\gamma}(t)$ gives (see [SWe, p. 171])

$$
\hat{F}_{\xi}^{\gamma}(t)=\int_{R^{n}} e^{-i x \cdot \xi} F_{x}^{\gamma}(t) d x=m_{\gamma}(t \xi) \hat{f}(\xi),
$$

where the multiplier

$$
m_{\gamma}(\xi)=|\xi|^{-\frac{n}{2}-\gamma} J_{\frac{n}{2}+\gamma}(|\xi|) .
$$

$J_{\frac{n}{2}+\gamma}$ is the Bessel function of order $\frac{n}{2}+\gamma$. (For more details about Bessel functions consult $[\mathbf{E}$ or $\mathbf{W}]$.) But since the multiplier $m_{\gamma}$ is well-defined for all complex $\gamma$, we can extend the mean $F^{\gamma}$ to these $\gamma$ 's.

The same letter $C$ will be used to denote various constants, not necessarily the same at each occurrence.

For some values of $\gamma$ the mean $F^{\gamma}$ has a special meaning.

If $\gamma=0$, then

$$
F_{x}^{0}(t)=C \int_{|y|<1} f(x-t y) d y=\frac{C}{|B(x, t)|} \int_{B(x, t)} f(y) d y
$$

the mean of $f$ over the ball $B(x, t)$ of radius $t$ with its centre in $x$.

If $\gamma=-1$, then

$$
F_{x}^{-1}(t)=C \int_{S^{n-1}} f(x-t y) d S(y)
$$

the mean of $f$ over the sphere of radius $t$ with its centre in $x . d S$ is the normalized Lebesgue measure on the unit sphere $S^{n-1}$.

Received by the editors February 12, 1987.

1980 Mathematics Subject Classification (1985 Revision). Primary 42B15; Secondary 44A30.

Key words and phrases. Mixed norm, spherical mean, wave equation. 
If $\gamma=-\frac{n-1}{2}$, then $u(x, t)=C t F_{x}^{-\frac{n-1}{2}}(t)$ solves the following Cauchy problem for the wave equation.

$$
\frac{\partial^{2} u}{\partial t^{2}}(x, t)=\sum_{i=1}^{n} \frac{\partial^{2} u}{\partial x_{i}{ }^{2}}(x, t)=\Delta_{x} u(x, t), \quad u(x, 0)=0, \frac{\partial u}{\partial t}(x, 0)=f(x)
$$

In this case the multiplier is given by

$$
m_{-\frac{n-1}{2}}(t \xi)=|t \xi|^{-\frac{1}{2}} J_{\frac{1}{2}}(|t \xi|)=C(\sin t|\xi|) / t|\xi| .
$$
data

If $\gamma=-\frac{n+1}{2}$, then $u(x, t)=C t F_{x}^{-\frac{n+1}{2}}(t)$ solves the wave equation with Cauchy

$$
u(x, 0)=f(x), \quad \frac{\partial u}{\partial t}(x, 0)=0 .
$$

The multiplier is then $m_{-\frac{n+1}{2}}(t \xi)=|t \xi|^{\frac{1}{2}} J_{-\frac{1}{2}}(|t \xi|)=C \cos t|\xi|$.

Estimates of spherical means which are related to the results in this paper can be found in [B1-B3, OB, PS, Sj1-Sj5, St2, STW, SWa and Str]. Related results of regularity properties of the solution of the wave equation are found in $[\mathbf{M a}, \mathbf{M i}$, $\mathbf{P r}, \mathbf{S s}, \mathbf{S t 2}$ and $\mathbf{S t r}]$. [Sj2] also contains an application to convergence of Fourier integrals.

2. Preliminaries. Let $C_{0}^{\infty}(\mathbf{R} \backslash\{0\})$ be the functions in $C^{\infty}(\mathbf{R})$ with compact support in $\mathbf{R} \backslash\{0\}$.

The operator $J^{\alpha}$ is defined by the relation $\left(J^{\alpha} \varphi\right)^{\wedge}(s)=\left(1+s^{2}\right)^{\alpha / 2} \hat{\varphi}(s)$, and the norm in the Bessel potential space $\mathscr{L}_{\alpha}^{p}(\mathbf{R})$ is defined by $\|\varphi\|_{\mathscr{L}_{\alpha}^{p}}=\left\|J^{\alpha} \varphi\right\|_{p}, 1 \leq$ $p \leq \infty$. Cf. [St1]. $\stackrel{\circ}{\mathscr{L}}_{\beta}^{2}(\mathbf{R} \backslash\{0\})$ is the closure of $C_{0}^{\infty}(\mathbf{R} \backslash\{0\})$ in the norm \|\|$_{\mathscr{L}_{\beta}^{2}}$. $\tilde{\mathscr{L}}_{\beta}^{2}(\mathbf{R} \backslash\{0\})$ is the space obtained by complex interpolation between $\stackrel{\mathscr{L}}{[\beta]}_{[\beta]}(\mathbf{R} \backslash\{0\})$ and $\stackrel{\circ}{\mathscr{L}}_{[\beta]+1}^{2}(\mathbf{R} \backslash\{0\})$, where $[\beta]$ is the integral part of $\beta,[\beta] \leq \beta<[\beta]+1$. The norm is denoted \|\|$_{\tilde{\mathscr{E}}_{\beta}^{2}}$ and coincides, by definition, with the norm of $\stackrel{\mathscr{L}}{\beta}_{\beta}^{2}$ when $\beta$ is an integer. Properties of the spaces $\stackrel{\mathscr{L}}{\beta}_{\beta}^{2}(\mathbf{R} \backslash\{0\})$ and $\tilde{\mathscr{L}}_{\beta}^{2}(\mathbf{R} \backslash\{0\})$ can be found in $[\mathbf{L M}]$.

$B M O(\mathbf{R})$ is the space of functions of bounded mean oscillation normed by

$$
\|\varphi\|_{B M O}=\sup _{I}\left[\left.|I|^{-1} \int_{I}|\varphi(t)-| I\right|^{-1} \int_{I} \varphi(s) d s \mid d t\right],
$$

where $I$ is a bounded interval. Cf. [St1, p. 164].

$\Lambda_{\delta}(\mathbf{R}), \delta>0$, is the Lipschitz space with norm

$$
\|\varphi\|_{\Lambda_{\delta}}=\|\varphi\|_{\infty}+\sup _{t, y} y^{k-\delta}\left|\frac{\partial^{k} u}{\partial y^{k}}(t, y)\right|
$$

where $u(t, y), t \in \mathbf{R}, y>0$, is the Poisson integral of $\varphi$ and $k$ is the smallest integer greater than $\delta$. See $[\mathbf{S t} \mathbf{1}]$.

The Hardy space $H^{p}\left(\mathbf{R}^{n}\right), 0<p \leq 1$, is defined to be the set of all temperate distributions $f$ such that

$$
\|f\|_{H^{p}}=\left\|\sup _{\varepsilon>0}\left|f * \psi_{\varepsilon}\right|\right\|_{p}<\infty
$$


where $\psi$ is some fixed element of $\mathscr{S}\left(\mathbf{R}^{n}\right)$ (the Schwartz class) with $\int \psi(x) d x \neq 0$ and $\psi_{\varepsilon}(x)=\varepsilon^{-n} \psi(x / \varepsilon)$. If $1<p<\infty, H^{p}$ is defined to be equal to $L^{p}$ with norm $\|f\|_{H^{p}}=\|f\|_{p}$. Cf. [FS].

Our results are the following.

THEOREM 1. If $n \geq 2, \frac{1}{p}+\frac{1}{p^{\prime}}=1$,

(i) $\gamma \geq-\frac{n+1}{2}$,

(ii) $\frac{n}{n+\frac{1}{2}+\gamma} \leq p \leq 2$,

(iii) $\beta=\frac{n+1}{2}+\gamma$, and

(iv) $\alpha=\frac{n}{p^{\prime}}+\frac{1}{2}+\gamma$

then

$$
\left(\int_{\mathbf{R}^{n}}\left\|\varphi F_{x}^{\gamma}\right\|_{\mathscr{L}_{\alpha}^{2}}^{2} d x\right)^{1 / 2} \leq C\|\varphi\|_{\tilde{\mathscr{L}}_{\beta}^{2}}\|f\|_{H^{p}}
$$

where $\varphi \in \tilde{\mathscr{L}}_{\beta}^{2}(\mathbf{R} \backslash\{0\})$ and $f \in C_{0}^{\infty}\left(\mathbf{R}^{n}\right) \cap H^{p}\left(\mathbf{R}^{n}\right)$. For $0 \leq \beta<\frac{1}{2}, \tilde{\mathscr{L}}_{\beta}^{2}(\mathbf{R} \backslash\{0\})$ and $\mathscr{L}_{\beta}^{2}(\mathbf{R})$ coincide. (1) is best possible in the sense that we cannot have $\alpha>$ $\frac{n}{p^{\prime}}+\frac{1}{2}+\gamma$.

REMARK 1. When $\gamma=-1$ and $\varphi$ is a fixed function in $C_{0}^{\infty}(\mathbf{R})$ with compact support in $(0, \infty)$ and $\|\varphi\|_{\tilde{\mathscr{L}}_{\beta}^{2}}$ is replaced by $C_{\varphi}$ in (1), then the result was obtained by P. Sjölin in $[\mathbf{S j 2}]$. In $[\mathbf{S j 4}]$ this was extended to a larger class of means, viz.

$$
\int_{S^{n-1}} f(x-t y) \rho(x, y) d S(y)
$$

where $\rho(x, y)$ satisfy certain differentiability properties.

COROLlary 1. Let $n \geq 2$ and $\gamma, p$ and $\beta$ satisfy (i) and (iii) of Theorem 1, $\varphi \in \tilde{\mathscr{L}}_{\beta}^{2}(\mathbf{R} \backslash\{0\})$ and $f \in C_{0}^{\infty}\left(\mathbf{R}^{n}\right) \cap H^{p}\left(\mathbf{R}^{n}\right)$.

If (v) $\frac{n}{n+\frac{1}{2}+\gamma} \leq p<\frac{n}{n+\gamma}$ and $q=-\left(\frac{n}{p^{\prime}}+\gamma\right)^{-1}$, then

$$
\left(\int_{\mathbf{R}^{n}}\left\|\varphi F_{x}^{\gamma}\right\|_{L^{q}}^{2} d x\right)^{\frac{1}{2}} \leq C\|\varphi\|_{\tilde{\mathscr{L}}_{\beta}^{2}}\|f\|_{H^{p}}
$$

If (vi) $p=\frac{n}{n+\gamma}$, then

$$
\left(\int_{\mathbf{R}^{n}}\left\|\varphi F_{x}^{\gamma}\right\|_{B M O}^{2} d x\right)^{\frac{1}{2}} \leq C\|\varphi\|_{\tilde{\mathscr{L}}_{\beta}}\|f\|_{H^{p}}
$$

If (vii) $\frac{n}{n+\gamma}<p \leq 2$ and $\delta=\frac{n}{p^{\prime}}+\gamma$, then

$$
\left(\int_{\mathbf{R}^{n}}\left\|\varphi F_{x}^{\gamma}\right\|_{\Lambda_{\delta}}^{2} d x\right)^{\frac{1}{2}} \leq C\|\varphi\|_{\tilde{\mathscr{L}}_{\beta}^{2}}\|f\|_{H^{p}}
$$

It is not possible to take $q>-\left(\frac{n}{p^{\prime}}+\gamma\right)^{-1}$ in (2). The BMO-norm in (3) cannot be replaced by a Lipschitz-norm and (4) is no longer true if $\delta>\frac{n}{p^{\prime}}+\gamma$.

REMARK 2. For $-1 \leq \gamma<0$, set

$$
f(x)= \begin{cases}|x|^{-n-\gamma}\left(\log \frac{1}{|x|}\right)^{-1}, & \text { if } 0<|x| \leq \frac{1}{2} \\ 0, & \text { otherwise. }\end{cases}
$$


Then $f \in L^{\frac{n}{n+\gamma}}\left(\mathbf{R}^{n}\right)$, but $F_{x}^{\gamma}(|x|)=\infty$. This shows that the BMO-norm in (3) is not replaceable by the sup-norm. If $p>1, \gamma>-1$ and $\varphi \in C_{0}^{\infty}(\mathbf{R} \backslash\{0\})$, then (1)-(4) is valid for $f \in L^{p}\left(\mathbf{R}^{n}\right)$. (The case $\gamma=-1$ is contained in [Sj2].) The details are carried out at the end of the proof of Corollary 1.

THEOREM 2. Assume that $n \geq 2, \varphi \in C_{0}^{\infty}(\mathbf{R} \backslash\{0\})$ and $f \in C_{0}^{\infty}\left(\mathbf{R}^{n}\right)$.

If (viii) $-\frac{n+1}{2} \leq \gamma \leq-1$, (ix) $\frac{n-1}{n+\gamma}<p \leq 2, p \leq r \leq p^{\prime}$, and (x) $0 \leq$ $\alpha<\frac{n-1}{p^{\prime}}+\gamma+1\left(\right.$ or $\left(\mathrm{ix}^{\prime}\right) \quad 2 \leq p<-\frac{n-1}{1+\gamma}=\left(\frac{n-1}{n+\gamma}\right)^{\prime}, r=p$, and $\left(\mathrm{x}^{\prime}\right) \quad 0 \leq \alpha<$ $\left.\frac{n-1}{p}+\gamma+1\right)$, then

$$
\left(\int_{\mathbf{R}^{n}}\left\|\varphi F_{x}^{\gamma}\right\|_{\mathscr{L}_{\alpha}^{p}}^{r} d x\right)^{\frac{1}{r}} \leq C_{\varphi}\|f\|_{p}
$$

If $\gamma$ satisfies (viii) and is equal to an integer or is such that $\frac{n+1}{2}+\gamma$ is equal to an integer, then the conclusion still holds, if $r=p>1$ and if $<$ is replaced by $\leq$ in (ix), (x), (ix') and $\left(\mathrm{x}^{\prime}\right)$.

REMARK 3 . We conjecture that Theorem 2 is still true if we also allow $p=\frac{n-1}{n+\gamma}$ in (ix) and equality in (x) and $\left(\mathrm{x}^{\prime}\right)$, since the conclusion holds for the endpoints $\gamma=-1$ and $\gamma=-\frac{n+1}{2}$ and for some values in between. -1 .

COROLlaRY 2. Let $n \geq 2, \varphi \in C_{0}^{\infty}(\mathbf{R} \backslash\{0\}), f \in C_{0}^{\infty}\left(\mathbf{R}^{n}\right)$ and $-\frac{n+1}{2} \leq \gamma \leq$ If (xi) $\frac{n-1}{n+\gamma}<p \leq \frac{n}{n+\gamma} \leq 2, p \leq q<-\left(\frac{n}{p^{\prime}}+\gamma\right)^{-1}, p \leq r \leq p^{\prime}$ (or (xi') $\left.2 \leq-\frac{n-2}{1+\gamma} \leq p<-\frac{n-1}{1+\gamma}, p \leq q<-\left(\frac{n-2}{p}+\gamma+1\right)^{-1}, r=p\right)$, then

$$
\left(\int_{\mathbf{R}^{n}}\left\|\varphi F_{x}^{\gamma}\right\|_{q}^{r} d x\right)^{\frac{1}{r}} \leq C_{\varphi}\|f\|_{p}
$$

If (xii) $\frac{n}{n+\gamma}<p \leq 2, p \leq r \leq p^{\prime}$ (or (xii') $2 \leq p<-\frac{n-2}{1+\gamma}, r=p$ ), then

$$
\left(\int_{\mathbf{R}^{n}}\left\|\varphi F_{x}^{\gamma}\right\|_{B M O}^{r} d x\right)^{\frac{1}{r}} \leq C_{\varphi}\|f\|_{p} \text {. }
$$

If (xiii) $\frac{n}{n+\gamma}<p \leq 2,0<\delta<\frac{n}{p^{\prime}}+\gamma, p \leq r \leq p^{\prime}$ (or (xiii') $2 \leq p<-\frac{n-2}{1+\gamma}$, $\left.0<\delta<\frac{n-2}{p}+\gamma+1, r=p\right)$, then

$$
\left(\int_{\mathbf{R}^{n}}\left\|\varphi F_{x}^{\gamma}\right\|_{\Lambda_{\delta}}^{r} d x\right)^{\frac{1}{r}} \leq C_{\varphi}\|f\|_{p}
$$

REMARK 4 . Here we also have the corresponding better estimates when $\gamma$ or $\frac{n+1}{2}+\gamma$ are integers. A combination of the methods and results of this paper with the estimates of $F_{x}^{\gamma}(1)$ given by Strichartz [Str] should give more mixed norm estimates.

COROllary 3. Let $\varphi \in C_{0}^{\infty}(\mathbf{R})$. Then it is possible to replace $\varphi(t)$ by $\varphi(t)|t|^{\eta}$ in (5)-(8), if

or

$$
\eta>\frac{n}{r^{\prime}}+\gamma, \quad p \leq 2
$$

$$
\eta>\frac{n-2}{p}+\gamma+1, \quad p \geq 2 \text {. }
$$


REMARK 5. Corollary 3 is contained in [Sj4, Theorem 4] in the case $\gamma=-1$ and $p \leq 2$, where it is also shown that the value $\frac{n}{r^{\prime}}-1$ is best possible.

EXAMPLES. The estimate (3), for $n=2, \gamma=-1$, can be seen as an endpoint result of Theorem 2 in [St2] and Theorem 1 in [B3].

Let $p=1$ and $\gamma=-\frac{1}{2}$. Then it is easy to see that the $H^{1}$-norm in (1) cannot be replaced by the $L^{1}$-norm. However, we have that $F_{x}^{-\frac{1}{2}}(t)$ maps $L^{1}\left(\mathbf{R}^{n}\right)$ to weak $L^{2}\left(\mathbf{R}^{n}\right)$ (since $\left(1-|y|^{2}\right)^{-\frac{1}{2}}$ is in weak $\left.L^{2}\left(\mathbf{R}^{n}\right)\right)$, i.e.

$$
\left|\left\{x ;\left|F_{x}^{-\frac{1}{2}}(t)\right|>\lambda\right\}\right| \leq C t^{-\frac{n}{2}}\left(\frac{\|f\|_{1}}{\lambda}\right)^{2} .
$$

This also shows that the estimate

$$
\left\|F^{-1}(1)\right\|_{1} \leq C\|f\|_{1}
$$

cannot be extended to

$$
\left\|F^{-1+i \mu}(1)\right\|_{1} \leq C(\mu)\|f\|_{1}, \quad \mu \in \mathbf{R},
$$

where $F^{-1+i \mu}(1)$ and $C(\mu)$ satisfy the hypothesis of the interpolation theorem of Stein [SWe, p. 205]. For it would then be possible to interpolate with

$$
\left\|F^{i \mu}(1)\right\|_{\infty} \leq C e^{\pi|\mu|}\|f\|_{1}, \quad \mu \in \mathbf{R}
$$

to get

$$
\left\|F^{-\frac{1}{2}}(1)\right\|_{2} \leq C\|f\|_{1},
$$

but this is false.

3. Proofs.

PROOF OF THEOREM 1 . We start with the case where $\alpha=0$ and prove a somewhat better estimate than (1). Let $\varphi \in C_{0}^{\infty}(\mathbf{R} \backslash\{0\}), f \in C_{0}^{\infty}\left(\mathbf{R}^{n}\right) \cap H^{p}\left(\mathbf{R}^{n}\right)$ and $\gamma=k+i \mu-\frac{n+1}{2}$, where $k$ is a nonnegative integer and $\mu \in \mathbf{R}$. With Fubini's theorem and Plancherel's identity we obtain

$$
\begin{aligned}
& \left(\int_{\mathbf{R}^{n}}\left\|\varphi F_{x}^{\gamma}\right\|_{\mathscr{L}_{0}^{2}}^{2} d x\right)^{\frac{1}{2}} \\
& \quad=\left(\int_{\mathbf{R}} \int_{\mathbf{R}^{n}}\left|\varphi(t) F_{x}^{\gamma}(t)\right|^{2} d x d t\right)^{\frac{1}{2}}=C\left(\int_{\mathbf{R}} \int_{\mathbf{R}^{n}}\left|\varphi(t) \hat{F}_{\xi}^{\gamma}(t)\right|^{2} d \xi d t\right)^{\frac{1}{2}} \\
& \quad=C\left(\left.\left.\int_{\mathbf{R}} \int_{\mathbf{R}^{n}}|\varphi(t)| t \xi\right|^{-\frac{n}{2}+\frac{n+1}{2}-k-i \mu} J_{\frac{n}{2}-\frac{n+1}{2}+k+i \mu}(|t \xi|) \hat{f}(\xi)\right|^{2} d \xi d t\right)^{\frac{1}{2}} \\
& \quad=C\left(\left.\left.\int_{\mathbf{R}} \int_{\mathbf{R}^{n}}|\varphi(t)| t \xi\right|^{\frac{1}{2}-k-i \mu} J_{-\frac{1}{2}+k+i \mu}(|t \xi|) \hat{f}(\xi)\right|^{2} d \xi d t\right)^{\frac{1}{2}} .
\end{aligned}
$$

The next step is to invoke the asymptotic estimate of Bessel functions for large arguments, i.e.

$$
\left|J_{-\frac{1}{2}+k+i \mu}(r)\right| \leq C_{k} r^{-\frac{1}{2}} e^{2 \pi|\mu|}
$$


where $r>0, k \in \mathbf{N}=\{0,1, \ldots\}$. See [W, pp. 217-218] or [B̈̈]. So (9) can be majorized by

$$
\begin{aligned}
C_{k} e^{2 \pi|\mu|} & \left(\left.\left.\int_{\mathbf{R}} \int_{\mathbf{R}^{n}}|\varphi(t)| t \xi\right|^{-k} \hat{f}(\xi)\right|^{2} d \xi d t\right)^{\frac{1}{2}} \\
= & C_{k} e^{2 \pi|\mu|}\left(\int_{\mathbf{R}}\left|\varphi(t) t^{-k}\right|^{2} d t\right)^{\frac{1}{2}}\left(\left.\left.\int_{\mathbf{R}^{n}}|\hat{f}(\xi)| \xi\right|^{-k}\right|^{2} d \xi\right)^{\frac{1}{2}} .
\end{aligned}
$$

Now we make use of the assumption that $\varphi^{(k)}(0)=0$ and Hardy's inequality, to see that

$$
\begin{gathered}
\left(\int_{\mathbf{R}}\left|\varphi(t) t^{-k}\right|^{2} d t\right)^{\frac{1}{2}} \leq C_{k}\left(\int_{\mathbf{R}}\left|\varphi^{\prime}(t) t^{-k+1}\right|^{2} d t\right)^{\frac{1}{2}} \\
\leq \ldots \leq C_{k}\left(\int_{\mathbf{R}}\left|\varphi^{(k)}(t)\right|^{2} d t\right)^{\frac{1}{2}} \leq C_{k}\|\varphi\|_{\mathscr{L}_{k}^{2}} .
\end{gathered}
$$

See [T, p. 262]. This gives

$$
\left(\int_{\mathbf{R}^{n}}\left\|\varphi F_{x}^{\gamma}\right\|_{\mathscr{L}_{0}^{2}}^{2} d x\right)^{\frac{1}{2}} \leq C_{k} e^{2 \pi|\mu|}\|\varphi\|_{\mathscr{L}_{k}^{2}}\left\|\hat{f}|\cdot|^{-k}\right\|_{2},
$$

for $k \in \mathbf{N}$. Consider the function $G_{x}^{\gamma}(t)$, defined by

$$
\left(G^{\gamma}(t)\right)^{\curlyvee}(\xi)=|\xi|^{k+i \mu} \hat{F}_{\xi}^{\gamma}(t) .
$$

Then (10) becomes

$$
\left(\int_{\mathbf{R}^{n}}\left\|\varphi G_{x}^{\gamma}\right\|_{2}^{2} d x\right)^{\frac{1}{2}} \leq C_{k} e^{2 \pi|\mu|}\|\varphi\|_{\mathscr{L}_{k}^{2}}\|\hat{f}\|_{2}=C_{k} e^{2 \pi|\mu|}\|\varphi\|_{\mathscr{L}_{k}^{2}}\|f\|_{2}
$$

where $k \in \mathbf{N}$. Using complex interpolation (see [CJ, Theorem 2]) between $k$ and $k+1$, we obtain

$$
\left(\int_{\mathbf{R}^{n}}\left\|\varphi G_{x}^{\gamma}\right\|_{2}^{2} d x\right)^{\frac{1}{2}} \leq C\|\varphi\|_{\tilde{\mathscr{L}}_{\beta}^{2}}\|f\|_{2}
$$

for $-\frac{n+1}{2}+k \leq \gamma \leq-\frac{n+1}{2}+k+1, \beta=\frac{n+1}{2}+\gamma$ and $k \in \mathbf{N}$, or equivalently

$$
\left(\int_{\mathbf{R}^{n}}\left\|\varphi F_{x}^{\gamma}\right\|_{\mathscr{L}_{0}^{2}}^{2} d x\right)^{\frac{1}{2}} \leq C\|\varphi\|_{\tilde{\mathscr{L}}_{\beta}^{2}}\left\|\hat{f}|\cdot|^{-\beta}\right\|_{2}
$$

for $\gamma \geq-\frac{n+1}{2}$ and $\beta=\frac{n+1}{2}+\gamma$. This is the improved inequality in the case $\alpha=0$.

We now consider the case $\alpha=\beta$, i.e. $p=2$ in (iv). Let $\varphi, f$ and $\gamma$ be as in the proof of the case $\alpha=0$ and set $D^{l}=\frac{d^{l}}{d t}$. In this proof we use the following

LEMMA 1. If $\nu \in \mathbf{C}, l \in \mathbf{N}, r>0$ and $\Re \nu \geq l-\frac{1}{2}$, then

$$
\left|r^{l} D^{l}\left(r^{-\nu} J_{\nu}(r)\right)\right| \leq C e^{3 \pi|\Im \nu|} .
$$

$C$ depends only on $\Re \nu$ and $l$. 
We postpone the proof of the lemma.

The $L^{2}\left(\mathscr{L}_{k}^{2}\right)$ norm of $\varphi F^{\gamma}, \gamma=k+i \mu-\frac{n+1}{2}$, is split into two $L^{2}$ norms.

$$
\left(\int_{\mathbf{R}^{n}}\left\|\varphi F_{x}^{\gamma}\right\|_{\mathscr{L}_{k}^{2}}^{2} d x\right)^{\frac{1}{2}} \leq C\left[\left(\int_{\mathbf{R}^{n}}\left\|\varphi F_{x}^{\gamma}\right\|_{2}^{2} d x\right)^{\frac{1}{2}}+\left(\int_{\mathbf{R}^{n}}\left\|D^{k}\left(\varphi F_{x}^{\gamma}\right)\right\|_{2}^{2} d x\right)^{\frac{1}{2}}\right]
$$

The first one is easily estimated ( $l=0$ in the lemma).

$$
\begin{aligned}
\left(\int_{\mathbf{R}^{n}}\left\|\varphi F_{x}^{\gamma}\right\|_{2}^{2} d x\right)^{\frac{1}{2}} & =C\left(\int_{\mathbf{R}} \int_{\mathbf{R}^{n}}\left|\varphi(t) \hat{F}_{\xi}^{-\frac{n+1}{2}+k+i \mu}(t)\right|^{2} d \xi d t\right)^{\frac{1}{2}} \\
& =C\left(\left.\left.\int_{\mathbf{R}} \int_{\mathbf{R}^{n}}|\varphi(t)| t \xi\right|^{\frac{1}{2}-k-i \mu} J_{-\frac{1}{2}+k+i \mu}(|t \xi|) \hat{f}(\xi)\right|^{2} d \xi d t\right)^{\frac{1}{2}} \\
& \leq C e^{3 \pi|\mu|}\|\varphi\|_{2}\|\hat{f}\|_{2} \leq C e^{3 \pi|\mu|}\|\varphi\|_{\mathscr{L}_{k}^{2}}\|f\|_{2}
\end{aligned}
$$

Set $B(r)=r^{-\frac{n}{2}-\gamma} J_{\frac{n}{2}+\gamma}(r)$ and estimate the second term again by Hardy's inequality.

$$
\begin{aligned}
\left(\int_{R^{n}}\right. & \left.\left\|D^{k}\left(\varphi F_{x}^{\gamma}\right)\right\|_{2}^{2} d x\right)^{\frac{1}{2}} \leq C\left(\int_{R^{n}}\left\|\sum_{l=0}^{k}\left(\begin{array}{c}
k \\
k-l
\end{array}\right) D^{k-l} \varphi D^{l} F_{x}^{\gamma}\right\|_{2}^{2} d x\right)^{\frac{1}{2}} \\
& \leq C \sum_{l=0}^{k}\left(\int_{R^{n}}\left\|D^{k-l} \varphi D^{l} F_{x}^{\gamma}\right\|_{2}^{2} d x\right)^{\frac{1}{2}} \\
& =C \sum_{l=0}^{k}\left(\int_{R}\left|D^{k-l} \varphi(t)\right|^{2} \int_{R^{n}}\left|D^{l} F_{x}^{\gamma}(t)\right|^{2} d x d t\right)^{\frac{1}{2}} \\
& =C \sum_{l=0}^{k}\left(\int_{R}\left|D^{k-l} \varphi(t)\right|^{2} \int_{R^{n}}\left|D^{l} \hat{F}_{\xi}^{\gamma}(t)\right|^{2} d \xi d t\right)^{\frac{1}{2}} \\
& =C \sum_{l=0}^{k}\left(\int_{R}\left|D^{k-l} \varphi(t)\right|^{2} \int_{R^{n}}\left|D^{l}(B(|t \xi|)) \hat{f}(\xi)\right|^{2} d \xi d t\right)^{\frac{1}{2}} \\
& =C \sum_{l=0}^{k}\left(\left.\left.\int_{R}\left|D^{k-l} \varphi(t)\right|^{2} \int_{R^{n}}|| \xi\right|^{l}(\operatorname{sgn} t)^{l}\left(D^{l} B\right)(|t \xi|) \hat{f}(\xi)\right|^{2} d \xi d t\right)^{\frac{1}{2}} \\
& =C \sum_{l=0}^{k}\left(\left.\int_{R}\left|D^{k-l} \varphi(t) t^{-l}\right|^{2} \int_{R^{n}}|t \xi|^{l}\left(D^{l} B\right)(|t \xi|) \hat{f}(\xi)\right|^{2} d \xi d t\right)^{\frac{1}{2}} \\
& \leq C e^{3 \pi|\mu|} \sum_{l=0}^{k}\left(\int_{R}\left|D^{k-l} \varphi(t) t^{-l}\right|^{2} \int_{R^{n}}|\hat{f}(\xi)|^{2} d \xi d t\right)^{\frac{1}{2}} \\
& =C e^{3 \pi|\mu|} \sum_{l=0}^{k}\left(\int_{R}\left|D^{k-l} \varphi(t) t^{-l}\right|^{2} d t\right)^{\frac{1}{2}}\|\hat{f}\|_{2} \\
\leq & C e^{3 \pi|\mu|}\left\|D^{k} \varphi\right\|_{2}\|f\|_{2} \leq C e^{3 \pi|\mu|}\|\varphi\|_{\mathscr{L}_{k}^{2}\|f\|_{2},}
\end{aligned}
$$


because $\Re\left(\frac{n}{2}+\gamma\right)=k-\frac{1}{2} \geq l-\frac{1}{2}$ and the condition in the lemma is fulfilled. So

$$
\left(\int_{\mathbf{R}^{n}}\left\|\varphi F_{x}^{\gamma}\right\|_{\mathscr{L}_{k}^{2}}^{2} d x\right)^{\frac{1}{2}} \leq C e^{3 \pi|\mu|}\|\varphi\|_{\mathscr{L}_{k}^{2}}\|f\|_{2}
$$

for $k \in \mathbf{N}$, and as before we interpolate between the $k$ 's. Using again the extension of Stein's interpolation theorem for the complex family $\varphi F^{\gamma}$ we get

$$
\left(\int_{\mathbf{R}^{n}}\left\|\varphi F_{x}^{\gamma}\right\|_{\mathscr{L}_{\beta}^{2}}^{2} d x\right)^{\frac{1}{2}} \leq C\|\varphi\|_{\tilde{\mathscr{L}}_{\beta}^{2}}\|f\|_{2},
$$

for $\gamma \geq-\frac{n+1}{2}$ and $\beta=\frac{n+1}{2}+\gamma$. See [CJ, Theorem 2]. For interpolation of the spaces $L^{2}\left(\mathscr{L}_{k}^{2}\right)$, see [BL, pp. 107 and 153]. This proves the theorem in the case $\alpha=\beta$.

We end up by interpolating between (11) and (12) with the following result.

$$
\left(\int_{\mathbf{R}^{n}}\left\|\varphi F_{x}^{\gamma}\right\|_{\mathscr{L}_{\alpha}^{2}}^{2} d x\right)^{\frac{1}{2}} \leq C\|\varphi\|_{\tilde{\mathscr{L}}_{\beta}^{2}}\left\|\hat{f}|\cdot|^{\alpha-\beta}\right\|_{2},
$$

where $0 \leq \alpha \leq \beta$. But from the boundedness of fractional integrals on $L^{p}$ spaces and its extension to $H^{p}$ spaces we also get that

$$
\left\|\hat{f}|\cdot|^{\alpha-\beta}\right\|_{2} \leq C\|f\|_{H^{p}},
$$

if

$$
\frac{1}{p}=\frac{1}{2}+\frac{\beta-\alpha}{n}=1+\frac{\frac{1}{2}+\gamma-\alpha}{n}, \quad \text { i.e } \quad \alpha=\frac{n}{p^{\prime}}+\frac{1}{2}+\gamma .
$$

See $[\mathbf{B L}$, p. 168 or $\mathbf{P}$, p. 50].

Hardy's inequality carries over from $C_{0}^{\infty}(\mathbf{R} \backslash\{0\})$ to $\stackrel{\circ}{\mathscr{L}}_{k}^{2}(\mathbf{R} \backslash\{0\})$ if the derivatives are to be understood in the weak sense. Consequently, the proof for $\varphi \in$ $C_{0}^{\infty}(\mathbf{R} \backslash\{0\})$ holds also for $\varphi \in \stackrel{\mathscr{L}}{k}_{k}^{2}(\mathbf{R} \backslash\{0\})$.

We continue with the proof of the identity $\tilde{\mathscr{L}}_{\beta}^{2}(\mathbf{R} \backslash\{0\})=\mathscr{L}_{\beta}^{2}(\mathbf{R}), 0 \leq \beta<\frac{1}{2}$. It is enough to show the identity $\stackrel{\mathscr{L}}{\beta}_{\beta}^{2}(\mathbf{R} \backslash\{0\})=\mathscr{L}_{\beta}^{2}(\mathbf{R})$, since $\tilde{\mathscr{L}}_{\beta}^{2}(\mathbf{R} \backslash\{0\})=$ $\stackrel{\mathscr{L}}{\beta}_{\beta}^{2}(\mathbf{R} \backslash\{0\})$ if $0 \leq \beta<\frac{1}{2}$ (see [LM, p. 64]).

Take a $\varphi$ in $\stackrel{\mathscr{L}}{\beta}_{\beta}^{2}(\mathbf{R} \backslash\{0\})$ and let $\left\{\varphi_{i}\right\}_{1}^{\infty}$ be a sequence in $C_{0}^{\infty}(\mathbf{R} \backslash\{0\})$ converging to $\varphi$. Extending the sequence to the whole real line by $\varphi_{i}(0)=0$, for all $i$, we obtain a sequence in $C_{0}^{\infty}(\mathbf{R})$ that converges to $\varphi$. Thus $\stackrel{\circ}{\mathscr{L}}_{\beta}^{2}(\mathbf{R} \backslash\{0\}) \subset \mathscr{L}_{\beta}^{2}(\mathbf{R})$.

Now take a $\varphi$ in $\mathscr{L}_{\beta}^{2}(\mathbf{R})$ and a sequence $\left\{\varphi_{i}\right\}_{1}^{\infty}$ in $C_{0}^{\infty}(\mathbf{R})$ such that $\left\|\varphi-\varphi_{i}\right\|_{\mathscr{L}_{\beta}^{2}} \rightarrow 0, n \rightarrow \infty$. From this sequence we shall construct another one in $C_{0}^{\infty}(\mathbf{R})$, with supports in $\mathbf{R} \backslash\{0\}$ and converging to $\varphi$ thus showing that $\mathscr{L}_{\beta}^{2}(\mathbf{R}) \subset \stackrel{\mathscr{L}}{\beta}_{\beta}^{2}(\mathbf{R} \backslash\{0\})$.

Let $\psi(t) \in C_{0}^{\infty}(\mathbf{R})$ be equal to 0 , if $|t|<\frac{1}{2}$, and equal to 1 , if $|t| \geq 1$. For given $\varepsilon>0$, choose $i$ such that $1 / i<\varepsilon$ and $\left\|\varphi-\varphi_{i}\right\|_{L_{\beta}^{2}}<\varepsilon / 2$. We claim that it is possible to choose, for each $i$, an $R=R(i)>1$ such that

$$
\left\|\varphi_{i}-\varphi_{i} \psi_{R(i)}\right\|_{\mathcal{L}_{\beta}^{2}}<\frac{1}{2 i}
$$


Here $\psi_{R}(t)=\psi(R t)$. Then $\left\{\varphi_{i} \psi_{R(i)}\right\}_{1}^{\infty}$ is the desired sequence, because

$$
\left\|\varphi-\varphi_{i} \psi_{R(i)}\right\|_{\mathscr{L}_{\beta}^{2}} \leq\left\|\varphi-\varphi_{i}\right\|_{\mathscr{L}_{\beta}^{2}}+\left\|\varphi_{i}-\varphi_{i} \psi_{R(i)}\right\|_{\mathscr{L}_{\beta}^{2}}<\frac{\varepsilon}{2}+\frac{1}{2 i}<\varepsilon
$$

Now to the proof of the claim. For $\beta=1$ we estimate the norm

$$
\begin{aligned}
\left\|\varphi_{i}-\varphi_{i} \psi_{R}\right\|_{\mathscr{L}_{1}^{2}} & =\left\|\varphi_{i}\left(1-\psi_{R}\right)\right\|_{\mathscr{L}_{1}^{2}} \\
& \leq\left\|\varphi_{i}\left(1-\psi_{R}\right)\right\|_{2}+\left\|\left(\varphi_{i}\left(1-\psi_{R}\right)\right)^{\prime}\right\|_{2} \\
& =\left\|\varphi_{i}\left(1-\psi_{R}\right)\right\|_{2}+\left\|\varphi_{i}\left(1-\psi_{R}\right)^{\prime}+\varphi_{i}^{\prime}\left(1-\psi_{R}\right)\right\|_{2} \\
& \leq\left\|\varphi_{i}\right\|_{\infty}\left\|1-\psi_{R}\right\|_{2}+\left\|\varphi_{i}\right\|_{\infty}\left\|\left(1-\psi_{R}\right)^{\prime}\right\|_{2}+\left\|\varphi_{i}^{\prime}\right\|_{\infty}\left\|1-\psi_{R}\right\|_{2} \\
& \leq\left(\left\|\varphi_{i}\right\|_{\infty}+\left\|\varphi_{i}^{\prime}\right\|_{\infty}\right)\left(2\left\|1-\psi_{R}\right\|_{2}+\left\|\left(1-\psi_{R}\right)^{\prime}\right\|_{2}\right) \\
& =\left(\left\|\varphi_{i}\right\|_{\infty}+\left\|\varphi_{i}^{\prime}\right\|_{\infty}\right)\left(2\left\|1-\psi_{R}\right\|_{2}+R\left\|(1-\psi)^{\prime}(R \cdot)\right\|_{2}\right) .
\end{aligned}
$$

A dilation gives that

$$
\begin{aligned}
\left\|\varphi_{i}-\varphi_{i} \psi_{R}\right\|_{\mathscr{L}_{1}^{2}} & \leq\left(\left\|\varphi_{i}\right\|_{\infty}+\left\|\varphi_{i}^{\prime}\right\|_{\infty}\right)\left(R^{-\frac{1}{2}} 2\|1-\psi\|_{2}+R^{\frac{1}{2}}\left\|(1-\psi)^{\prime}\right\|_{2}\right) . \\
& \leq R^{\frac{1}{2}} 2\left(\left\|\varphi_{i}\right\|_{\infty}+\left\|\varphi_{i}^{\prime}\right\|_{\infty}\right)\|1-\psi\|_{\mathscr{L}_{1}^{2}},
\end{aligned}
$$

if we choose $R \geq 1$. Setting $\psi_{1}=1-\psi$ this can be rewritten as

$$
\left\|\varphi_{i} \psi_{1}(R \cdot)\right\|_{\mathscr{L}_{1}^{2}} \leq R^{\frac{1}{2}} 2\left(\left\|\varphi_{i}\right\|_{\infty}+\left\|\varphi_{i}^{\prime}\right\|_{\infty}\right)\left\|\psi_{1}\right\|_{\mathscr{L}_{1}^{2}} .
$$

For $\beta=0$, we have

$$
\begin{aligned}
\left\|\varphi_{i}-\varphi_{i} \psi_{R}\right\|_{\mathscr{L}_{0}^{2}} & =\left\|\varphi_{i}\left(1-\psi_{R}\right)\right\|_{2} \leq\left\|\varphi_{i}\right\|_{\infty}\left\|1-\psi_{R}\right\|_{2} \\
& =\left\|\varphi_{i}\right\|_{\infty} R^{-\frac{1}{2}}\|1-\psi\|_{2} \leq R^{-\frac{1}{2}} 2\left(\left\|\varphi_{i}\right\|_{\infty}+\left\|\varphi_{i}^{\prime}\right\|_{\infty}\right)\|1-\psi\|_{2}
\end{aligned}
$$

or equivalently

$$
\left\|\varphi_{i} \psi_{1}(R \cdot)\right\|_{\mathscr{L}_{0}^{2}} \leq R^{-\frac{1}{2}} 2\left(\left\|\varphi_{i}\right\|_{\infty}+\left\|\varphi_{i}^{\prime}\right\|_{\infty}\right)\left\|\psi_{1}\right\|_{\mathscr{L}_{0}^{2}}
$$

Interpolating between (13) and (14) yields

$$
\left\|\varphi_{i} \psi_{1}(R \cdot)\right\|_{\mathscr{L}_{\beta}^{2}} \leq R^{\beta-\frac{1}{2}} 2\left(\left\|\varphi_{i}\right\|_{\infty}+\left\|\varphi_{i}^{\prime}\right\|_{\infty}\right)\left\|\psi_{1}\right\|_{\mathscr{L}_{\beta}^{2}}
$$

Since $0 \leq \beta<\frac{1}{2}$ it is possible to choose $R>1$ so that the right-hand side of (15) becomes less than $\frac{1}{2 i}$.

We finish the proof of Theorem 1 by showing that it is impossible to have $\alpha>$ $\frac{n}{p^{\prime}}+\frac{1}{2}+\gamma$ in (1).

Take a fixed $a \geq 1$. Set $T_{t}^{\gamma} f(x)=F_{x}^{\gamma}(t)$ and $g_{a}(x)=g(a x)$. Computing the Fourier transform of $g_{a}$ yields $\widehat{\left(g_{a}\right)}(\xi)=a^{-n} \hat{g}(\xi / a)$. With these identities we get

$$
\begin{aligned}
\left(T_{t}^{\gamma}\left(f_{a}\right)\right)^{\curlyvee}(\xi) & =m_{\gamma}(t \xi) \widehat{\left(f_{a}\right)}(\xi)=m_{\gamma}(t \xi) a^{-n} \hat{f}(\xi / a) \\
& =m_{\gamma}\left(a t \xi a^{-1}\right) a^{-n} \hat{f}(\xi / a)=a^{-n}\left(T_{a t}^{\gamma} f\right)^{\curlyvee}(\xi / a)=\left(\left(T_{a t}^{\gamma} f\right)_{a}\right)^{\Upsilon}(\xi) .
\end{aligned}
$$

That is $T_{t}^{\gamma}\left(f_{a}\right)(x)=\left(T_{a t}^{\gamma} f\right)(a x)$. Applying (1) gives

$$
\begin{aligned}
\left(\int_{\mathbf{R}^{n}}\left\|\varphi_{a} T^{\gamma}\left(f_{a}\right)(x)\right\|_{\mathscr{L}_{\alpha}^{2}}^{2} d x\right)^{\frac{1}{2}} & =\left(\int_{\mathbf{R}^{n}}\left\|\varphi(a \cdot)\left(T_{a}^{\gamma} f\right)_{a}(x)\right\|_{\mathscr{L}_{\alpha}^{2}}^{2} d x\right)^{\frac{1}{2}} \\
& \leq C\|\varphi(a \cdot)\|_{\tilde{\mathscr{L}}_{\beta}^{2}}\left\|f_{a}\right\|_{H^{p}} .
\end{aligned}
$$


Putting $\psi_{a}(t)=\psi(a t)=T_{a t}^{\gamma} f$, then

$$
\begin{aligned}
& \left\|\varphi_{a} \psi_{a}\right\|_{\mathscr{L}_{\alpha}^{2}}=\left(\int_{\mathbf{R}}\left|\left((\varphi \psi)_{a}\right)^{\uparrow}(s)\right|^{2}\left(1+s^{2}\right)^{\alpha} d s\right)^{\frac{1}{2}} \\
& =\left(\int_{\mathbf{R}}\left|a^{-1}(\widehat{\varphi \psi})(s / a)\right|^{2}\left(1+s^{2}\right)^{\alpha} d s\right)^{\frac{1}{2}} \\
& =a^{-1}\left(\int_{\mathbf{R}}|\widehat{(\varphi \psi)}(t)|^{2}\left(1+a^{2} t^{2}\right)^{\alpha} a d t\right)^{\frac{1}{2}} \\
& \geq a^{-\frac{1}{2}}\left(\int_{\mathbf{R}}|\widehat{(\widehat{\psi})}(t)|^{2}\left(a^{2} t^{2}\right)^{\alpha} d t\right)^{\frac{1}{2}} \\
& =a^{-\frac{1}{2}+\alpha}\left(\int_{\mathbf{R}}|(\widehat{\varphi \psi})(t)|^{2} t^{2 \alpha} d t\right)^{\frac{1}{2}} .
\end{aligned}
$$

A change of variables in the integral defining the $H^{p}$-norm gives $\left\|f_{a}\right\|_{H^{p}}=a^{-\frac{n}{p}}\|f\|_{H^{p}}$.

We introduce the space

$$
\stackrel{\circ}{\mathscr{L}}_{\beta}^{2}(\mathbf{R} \backslash\{0\})=\left\{\varphi ; \varphi \in \stackrel{\circ}{\mathscr{L}}_{\beta}^{2}(\mathbf{R} \backslash\{0\}),|\cdot|{ }^{[\beta]-\beta} D^{[\beta]} \varphi \in L^{2}(\mathbf{R} \backslash\{0\})\right\}
$$

with norm

$$
\|\varphi\|_{\mathscr{\mathscr { L }}_{\beta}^{2}}=\|\varphi\|_{\mathscr{L}_{\beta}^{2}}+\left\||\cdot|^{[\beta]-\beta} D^{[\beta]} \varphi\right\|_{2} .
$$

With this space we have a description of $\tilde{\mathscr{L}}_{\beta}^{2}(\mathbf{R} \backslash\{0\})$, viz.

$$
\tilde{\mathscr{L}}_{\beta}^{2}(\mathbf{R} \backslash\{0\})= \begin{cases}\check{\mathscr{L}}_{\beta}^{2}(\mathbf{R} \backslash\{0\}), & \text { if } \beta-[\beta]=\frac{1}{2}, \\ \stackrel{\mathscr{L}}{\beta}_{\beta}^{2}(\mathbf{R} \backslash\{0\}), & \text { otherwise. }\end{cases}
$$

See [LM, p. 66]. Thus $\stackrel{\circ}{\mathscr{L}}_{\beta}^{2}(\mathbf{R} \backslash\{0\}) \subset \tilde{\mathscr{L}}_{\beta}^{2}(\mathbf{R} \backslash\{0\})$ and we get that $\|\varphi(a \cdot)\|_{\tilde{\mathscr{Z}}_{\beta}^{2}} \leq\|\varphi(a \cdot)\|_{\mathscr{L}_{\beta}^{2}}+\left\||\cdot|^{[\beta]-\beta} D^{[\beta]} \varphi(a \cdot)\right\|_{2}$

$$
\begin{aligned}
& =\left(\int_{\mathbf{R}}\left|a^{-1} \hat{\varphi}(s / a)\right|^{2}\left(1+s^{2}\right)^{\beta} d s\right)^{\frac{1}{2}}+\left(\left.\left.\int_{\mathbf{R}}|| t\right|^{[\beta]-\beta} a^{[\beta]}\left(D^{[\beta]} \varphi\right)(a t)\right|^{2} d t\right)^{\frac{1}{2}} \\
& =\left(\int_{\mathbf{R}}\left|a^{-1} \hat{\varphi}(u)\right|^{2}\left(1+a^{2} u^{2}\right)^{\beta} a d u\right)^{\frac{1}{2}}+\left(\left.\left.\int_{\mathbf{R}}\left|a^{\beta}\right| v\right|^{[\beta]-\beta}\left(D^{[\beta]} \varphi\right)(v)\right|^{2} a^{-1} d v\right)^{\frac{1}{2}} \\
& \leq a^{-\frac{1}{2}}\left(\int_{\mathbf{R}}|\hat{\varphi}(u)|^{2}\left(a^{2}\left(1+u^{2}\right)\right)^{\beta} d u\right)^{\frac{1}{2}}+a^{-\frac{1}{2}+\beta}\left(\left.\left.\int_{\mathbf{R}}|| v\right|^{[\beta]-\beta}\left(D^{[\beta]} \varphi\right)(v)\right|^{2} d v\right)^{\frac{1}{2}} \\
& =a^{-\frac{1}{2}+\beta}\|\varphi\|_{\mathcal{L}_{\beta}^{2}} .
\end{aligned}
$$

Summing up the estimates gives

$$
\begin{aligned}
\left(\int_{\mathbf{R}^{n}}\left\|\varphi_{a}\left(T_{a}^{\gamma} f\right)_{a}(x)\right\|_{\mathscr{L}_{\alpha}^{2}}^{2} d x\right)^{\frac{1}{2}} & =a^{-\frac{n}{2}}\left(\int_{\mathbf{R}^{n}}\left\|\varphi_{a} T_{a}^{\gamma} f(x)\right\|_{\mathscr{L}_{\alpha}^{2}}^{2} d x\right)^{\frac{1}{2}} \\
& \geq a^{-\frac{n}{2}} a^{-\frac{1}{2}+\alpha} C=C a^{-\frac{n}{2}-\frac{1}{2}+\alpha} .
\end{aligned}
$$


$\checkmark$ does not depend on $a$. But we also have that

$\left(\int_{\mathbf{R}^{n}}\left\|\varphi_{a} T^{\gamma}\left(f_{a}\right)(x)\right\|_{\mathscr{L}_{\alpha}^{2}}^{2} d x\right)^{\frac{1}{2}} \leq C\left\|\varphi_{a}\right\|_{\tilde{\mathscr{L}}_{\beta}^{2}}\left\|f_{a}\right\|_{H^{p}} \leq a^{-\frac{1}{2}+\beta} C a^{-\frac{n}{p}} C=C a^{-\frac{1}{2}+\beta-\frac{n}{p}}$ $\varphi \in \stackrel{\circ}{\mathscr{L}}_{\beta}^{2}$. With the $a$ 's on the left-hand side

$$
a^{-\frac{n}{2}-\frac{1}{2}+\alpha+\frac{1}{2}-\beta+\frac{n}{p}}=a^{\alpha-\beta-\frac{n}{2}+\frac{n}{p}} \leq C
$$

or

$$
\alpha-\beta-\frac{n}{2}+\frac{n}{p} \leq 0
$$

because $a \geq 1$. But this implies

$$
\alpha \leq \beta+\frac{n}{2}-\frac{n}{p}=\frac{n+1}{2}+\gamma+\frac{n}{2}-\frac{n}{p}=\frac{n}{p^{\prime}}+\frac{1}{2}+\gamma .
$$

So it is impossible to have

$$
\alpha>\frac{n}{p^{\prime}}+\frac{1}{2}+\gamma
$$

This ends the proof of Theorem 1.

PROOF OF LEMMA 1 . We use the formula

$$
2 D^{1} J_{\nu}(r)=J_{\nu-1}(r)-J_{\nu+1}(r)
$$

and the fact that

$$
\left|J_{\nu}(r)\right| \leq C e^{2 \pi|\Im \nu|} r^{-\frac{1}{2}}
$$

if $\Re \nu \geq-\frac{1}{2}$ and $r>0$. Here $C$ depends only on $\Re \nu$. See [W, pp. 45 and 217-218] or [Bö]. The first identity repeated $j$ times gives

$$
D^{j} J_{\nu}(r)=\sum_{i=-j}^{j} a_{i} J_{\nu+i}(r)
$$

So

$$
\begin{gathered}
r^{l} D^{l}\left(r^{-\nu} J_{\nu}(r)\right)=r^{l} \sum_{j=0}^{l} b_{j} r^{-\nu-(l-j)} D^{j} J_{\nu}(r)=r^{l} \sum_{j=0}^{l} b_{j} r^{-\nu-l+j} \sum_{i=-j}^{j} a_{i} J_{\nu+i}(r) \\
=r^{l} \sum_{j=0}^{l} \sum_{i=-j}^{j} b_{j} a_{i} r^{-l+j+i} r^{-\nu-i} J_{\nu+i}(r)=\sum_{j=0}^{l} \sum_{i=-j}^{j} b_{j} a_{i} r^{j+i} r^{-(\nu+i)} J_{\nu+i}(r)
\end{gathered}
$$

The case $0 \leq r \leq 1: r^{\nu+i} \leq 1$, since $j+i \geq 0$ and

$$
\left|r^{-(\nu+i)} J_{\nu+i}(r)\right| \leq C e^{2 \pi|\Im \nu|}
$$

since $\Re(\nu+i) \geq \Re \nu-j \geq \Re \nu-l \geq-\frac{1}{2}$ and as a consequence the double sum is bounded by $C e^{3 \pi|\Im \nu|}$, because $b_{j}$ have only polynomial growth in $\Im \nu$.

The case $r \geq 1$. We have that

$$
\left|r^{-(\nu+i)} J_{\nu+i}(r)\right| \leq C e^{2 \pi|\Im \nu|} r^{-\frac{1}{2}-\nu-i}
$$


since $\Re(\nu+i) \geq-\frac{1}{2}$. Therefore,

$$
\begin{aligned}
\left|r^{l} D^{l}\left(r^{-\nu} J_{\nu}(r)\right)\right| & \leq \sum_{j=0}^{l}\left|b_{j} a_{i} r^{-\nu+j}\right| C e^{2 \pi|\Im \nu|} r^{-\frac{1}{2}} \\
& \leq C e^{3 \pi|\Im \nu|} \sum_{j=0}^{l} r^{-\Re \nu+j-\frac{1}{2}} \leq C e^{3 \pi|\Im \nu|}
\end{aligned}
$$

because $-\Re \nu+j-\frac{1}{2} \leq-\Re \nu+l-\frac{1}{2} \leq 0$. This shows the lemma.

PROOF OF COROLLARY 1. If $\frac{1}{q}=\frac{1}{2}-\alpha$ and $0 \leq \alpha<\frac{1}{2}$, then $\mathscr{L}_{\alpha}^{2}(\mathbf{R}) \subset L^{q}(\mathbf{R})$ with corresponding norm inequalities. Thus (2) follows if

$$
0 \leq \frac{n}{p^{\prime}}+\frac{1}{2}+\gamma<\frac{1}{2} \text { and } \frac{1}{q}=\frac{1}{2}-\left(\frac{n}{p^{\prime}}+\frac{1}{2}+\gamma\right),
$$

but this is $(v)$.

$\mathscr{L}_{\frac{1}{2}}^{2}(\mathbf{R})$ is continuously embedded in $B M O(\mathbf{R})$, i.e.

$$
\frac{1}{2}=\frac{n}{p^{\prime}}+\frac{1}{2}+\gamma
$$

which is (vi) and then (3) follows.

If

$$
\delta=\alpha-\frac{1}{2} \quad \text { and } \quad \alpha=\frac{n}{p^{\prime}}+\frac{1}{2}+\gamma>\frac{1}{2}
$$

then $\mathscr{L}_{\alpha}^{2}(\mathbf{R}) \subset \Lambda_{\delta}(\mathbf{R})$ and as a consequence we have (4) if (vii) holds.

Compare with the proof of Corollary 2.

In the homogeneity argument showing the necessity of

$$
\alpha=\frac{n}{p^{\prime}}+\frac{1}{2}+\gamma
$$

in (1), we used that

$$
\left\|\varphi_{a}\right\|_{\mathscr{L}_{\alpha}^{2}} \geq C a^{-\frac{1}{2}+\alpha} .
$$

Here $C$ is independent of $a$. In the same way it is easy to see that (2), (3) and (4) can not be improved using

$$
\left\|\varphi_{a}\right\|_{q}=a^{-\frac{1}{q}}\|\varphi\|_{q}
$$

and, for $0<\delta<1$,

$$
\begin{aligned}
\left\|\varphi_{a}\right\|_{\Lambda_{\delta}} & =\left\|\varphi_{a}\right\|_{\infty}+\sup _{|t|>0} \frac{\left\|\varphi_{a}(u+t)-\varphi_{a}(u)\right\|_{\infty}}{|t|^{\delta}} \\
& \geq \sup _{|t|>0} \frac{\|\varphi(a u+a t)-\varphi(a u)\|_{\infty}}{|t|^{\delta}}=a^{\delta} \sup _{|t|>0} \frac{\|\varphi(v+a t)-\varphi(v)\|_{\infty}}{|a t|^{\delta}}=C a^{\delta} .
\end{aligned}
$$

When $\delta \geq 1$ the argument is similar but involves higher order differences. See [St1, Chapter V, §4].

Next we prove the extension of $f$ to $L^{p}\left(\mathbf{R}^{n}\right)$ if $p>1, \gamma>-1$ and $\varphi \in$ $C_{0}^{\infty}(\mathbf{R} \backslash\{0\})$.

Assume that supp $\varphi \subset(0, \infty)$ and $f \in L^{p}\left(\mathbf{R}^{n}\right)$. Let $\left\{f_{k}\right\}_{1}^{\infty}$ be a sequence of functions in $C_{0}^{\infty}\left(\mathbf{R}^{n}\right)$ converging to $f$ in $L^{p}\left(\mathbf{R}^{n}\right)$, as $k \rightarrow \infty$, and let $F_{x, k}^{\gamma}(t)$ be 
the mean of $f_{k}$. Estimating the Fourier transform of $\varphi(t)\left(F_{x}^{\gamma}(t)-F_{x, k}^{\gamma}(t)\right)$ in the $t$-variable gives

$$
\begin{aligned}
& \left|\left(\varphi\left(F_{x}^{\gamma}-F_{x, k}^{\gamma}\right)\right)^{-}(s)\right|=\left|\int_{\mathbf{R}} e^{-i s t} \varphi(t)\left(F_{x}^{\gamma}(t)-F_{x, k}^{\gamma}(t)\right) d t\right| \\
& \quad=\left|\int_{0}^{\infty} e^{-i s t} \varphi(t) \int_{\mathbf{R}^{n}}\left(f(x-t y)-f_{k}(x-t y)\right)\left(1-|y|^{2}\right)_{+}^{\gamma} d y d t\right| \\
& \quad=\left|\int_{0}^{\infty} e^{-i s t} \varphi(t) \int_{0}^{1} \int_{S^{n-1}}\left(f-f_{k}\right)\left(x-t r y^{\prime}\right)\left(1-r^{2}\right)^{\gamma} d S\left(y^{\prime}\right) r^{n-1} d r d t\right| \\
& \quad=\left.\left|\int_{0}^{1} r^{n-1}\left(1-r^{2}\right)^{\gamma} \int_{\mathbf{R}^{n}}\left(f-f_{k}\right)(x-r y) e^{-i s|y|} \varphi(|y|)\right| y\right|^{1-n} d y d r \mid .
\end{aligned}
$$

Set $\varphi_{1}(y)=\varphi(|y|)|y|^{1-n}$ and change variables, $y=\frac{z}{r}$, in the inner integral. Then

$$
\begin{aligned}
\left|\left(\varphi\left(F_{x}^{\gamma}-F_{x, k}^{\gamma}\right)\right)^{-}(s)\right| & \leq \int_{0}^{1} \int_{\mathbf{R}^{n}}\left|\varphi_{1}\left(\frac{z}{r}\right)\right|\left|\left(f-f_{k}\right)(x-z)\right| d z\left(1-r^{2}\right)^{\gamma} \frac{d r}{r} \\
& \leq \int_{0}^{1}\left\|\varphi_{1}\left(\frac{\dot{r}}{r}\right)\right\|_{p^{\prime}}\left(1-r^{2}\right)^{\gamma} \frac{d r}{r}\left\|f-f_{k}\right\|_{p}
\end{aligned}
$$

by Hölder's inequality. Here

$$
\int_{0}^{1}\left\|\varphi_{1}\left(\frac{\dot{r}}{r}\right)\right\|_{p^{\prime}}\left(1-r^{2}\right)^{\gamma} \frac{d r}{r} \leq C,
$$

if $p>1, \gamma>-1$, and $\left\|f-f_{k}\right\|_{p} \rightarrow 0$ as $k \rightarrow \infty$. An application of Fatou's lemma now shows that

$$
\begin{aligned}
\left(\int_{\mathbf{R}^{n}}\left\|\varphi F_{x}^{\gamma}\right\|_{\mathscr{L}_{\alpha}^{2}}^{2} d x\right)^{\frac{1}{2}} & =\left(\int_{\mathbf{R}^{n}} \int_{\mathbf{R}}\left|\left(\varphi F_{x}^{\gamma}\right)^{\curlyvee}(s)\right|^{2}\left(1+s^{2}\right)^{\alpha} d s d x\right)^{\frac{1}{2}} \\
& =\left(\int_{\mathbf{R}^{n}} \int_{\mathbf{R}} \underline{\lim }\left|\left(\varphi F_{x, k}^{\gamma}\right)^{\curlyvee}(s)\right|^{2}\left(1+s^{2}\right)^{\alpha} d s d x\right)^{\frac{1}{2}} \\
& \leq \underline{\lim }\left(\int_{\mathbf{R}^{n}} \int_{\mathbf{R}}\left|\left(\varphi F_{x, k}^{\gamma}\right)^{\curlyvee}(s)\right|^{2}\left(1+s^{2}\right)^{\alpha} d s d x\right)^{\frac{1}{2}} \\
& \leq \underline{\lim } C\left\|f_{k}\right\|_{p}=C\|f\|_{p}
\end{aligned}
$$

and (1)-(4) can be extended to $f \in L^{p}\left(\mathbf{R}^{n}\right)$.

This also applies to $\varphi$ such that $\operatorname{supp} \varphi \subset(-\infty, 0)$, and therefore all $\varphi \in$ $C_{0}^{\infty}(\mathbf{R} \backslash\{0\})$ by splitting the support in two.

PROOF OF THEOREM 2. The proof is divided into two parts. In the first one we prove (5) in the case $\alpha=0$. The second part contains an interpolation argument, where the result proved in the first part is interpolated with the $L^{2}$ case of Theorem 1.

Assume that $\varphi \in C_{0}^{\infty}(\mathbf{R} \backslash\{0\})$ and $f \in C_{0}^{\infty}\left(\mathbf{R}^{n}\right) \cap H^{p}\left(\mathbf{R}^{n}\right)$. Consider the mean for $\gamma=-1+\varepsilon+i \mu, 0<\varepsilon<1, \mu \in \mathbf{R}$.

$$
F_{x}^{-1+\varepsilon+i \mu}(t)=\frac{C 2^{-\varepsilon-i \mu}}{\Gamma(\varepsilon+i \mu)} \int_{|y|<1}\left(1-|y|^{2}\right)^{-1+\varepsilon+i \mu} f(x-t y) d y .
$$


$C$ depends on the dimension $n$ only. Taking $t=1$, the $L^{1}\left(\mathbf{R}^{n}\right)$ norm of the mean can be estimated:

$$
\begin{aligned}
\left\|F^{-1+\varepsilon+i \mu}(1)\right\|_{1} & =\int_{\mathbf{R}^{n}}\left|\frac{C 2^{-\varepsilon-i \mu}}{\Gamma(\varepsilon+i \mu)} \int_{|y|<1}\left(1-|y|^{2}\right)^{-1+\varepsilon+i \mu} f(x-y) d y\right| d x \\
& \leq \frac{C 2^{-\varepsilon}}{|\Gamma(\varepsilon+i \mu)|} \int_{|y|<1}\left(1-|y|^{2}\right)^{-1+\varepsilon} \int_{\mathbf{R}^{n}}|f(x-y)| d x d y \\
& \leq \frac{C 2^{-\varepsilon}}{|\Gamma(\varepsilon+i \mu)|} \int_{|y|<1}\left(1-|y|^{2}\right)^{-1+\varepsilon} d y\|f\|_{1} \\
& \leq C_{\varepsilon}|\Gamma(\varepsilon+i \mu)|^{-1}\|f\|_{1}=C_{\varepsilon} e^{\pi|\mu|}\|f\|_{1} .
\end{aligned}
$$

The estimate of the gamma function can be found in [E, Volume 1, p. 47].

We continue with the $L^{2}$ estimate of the mean when $\gamma=-\frac{n+1}{2}+i \mu$ and $t=1$. In this case the multiplier

$$
m_{-\frac{n+1}{2}+i \mu}(\xi)=|\xi|^{\frac{1}{2}-i \mu} J_{-\frac{1}{2}+i \mu}(|\xi|)
$$

and as we have seen in Lemma 1 it can be estimated, i.e.

$$
\left|m_{-\frac{n+1}{2}+i \mu}(\xi)\right| \leq C e^{3 \pi|\mu|} .
$$

$C$ depends only on the dimension $n$. Therefore by Plancherel's identity

$$
\begin{aligned}
\left\|F^{-\frac{n+1}{2}+i \mu}(1)\right\|_{2} & =C\left\|\hat{F}^{-\frac{n+1}{2}+i \mu}(1)\right\|_{2}=C\left\|m_{-\frac{n+1}{2}+i \mu} \hat{f}\right\|_{2} \\
& \leq C e^{3 \pi|\mu|}\|\hat{f}\|_{2}=C e^{3 \pi|\mu|}\|f\|_{2} .
\end{aligned}
$$

The operator $F^{\gamma}(1)$ is of "admissible" growth, so we can perform the complex interpolation of Stein (see [SWe, p. 205]) to get the following:

$$
\left\|F^{\gamma}(1)\right\|_{p} \leq C\|f\|_{p}
$$

where $-\frac{n+1}{2} \leq \gamma \leq-1+\varepsilon$ and $p=\frac{n-1+2 \varepsilon}{n+\gamma+\varepsilon}$. By duality we also have (16) if $p^{\prime}=\frac{n-1+2 \varepsilon}{n+\gamma+\varepsilon}$. Now the standard dilation argument shows that (16) can be replaced with

$$
\left\|F^{\gamma}(t)\right\|_{p} \leq C\|f\|_{p} .
$$

The constant $C$ does not depend on the variable $t$. Now by Fubini's theorem

$$
\begin{aligned}
\left(\int_{\mathbf{R}^{n}}\left\|\varphi F_{x}^{\gamma}\right\|_{p}^{p} d x\right)^{\frac{1}{p}} & =\left(\int_{\mathbf{R}^{n}} \int_{\mathbf{R}}\left|\varphi(t) F_{x}^{\gamma}(t)\right|^{p} d t d x\right)^{\frac{1}{p}} \\
& =\left(\int_{\mathbf{R}}|\varphi(t)|^{p} \int_{\mathbf{R}^{n}}\left|F_{x}^{\gamma}(t)\right|^{p} d x d t\right)^{\frac{1}{p}} \\
& \leq\left(\int_{\mathbf{R}}|\varphi(t)|^{p} C^{p}\|f\|_{p}^{p} d t\right)^{\frac{1}{p}}=C\|\varphi\|_{p}\|f\|_{p} .
\end{aligned}
$$


Take $\varphi, f, \varepsilon$, and $\mu$ as before and define $a_{+}=\max (a, 0)$. This gives

$$
\left\|\varphi F_{x}^{\varepsilon-1+i \mu}\right\|_{1}=\int_{\mathbf{R}}\left|\varphi(t) \frac{2^{-\varepsilon+1-i \mu}(2 \pi)^{-\frac{n}{2}}}{\Gamma(\varepsilon+i \mu)} \int_{\mathbf{R}^{n}}\left(1-|y|^{2}\right)_{+}^{\varepsilon-1+i \mu} f(x-t y) d y\right| d t
$$

A change of variable $z=t y$ makes this equal to

$$
\left.\frac{2^{1-\varepsilon}(2 \pi)^{-\frac{n}{2}}}{|\Gamma(\varepsilon+i \mu)|} \int_{\mathbf{R}}\left|\varphi(t) \int_{\mathbf{R}^{n}}\left(1-\left|\frac{z}{t}\right|^{2}\right)_{+}^{\varepsilon-1+i \mu} f(x-z)\right| t\right|^{-n} d z \mid d t
$$

Again using the asymptotic expansion of the gamma function and Fubini's theorem we get that

$$
\left\|\varphi F_{x}^{\varepsilon-1+i \mu}\right\|_{1} \leq C_{\varepsilon} e^{3 \pi|\mu|} \int_{\mathbf{R}^{n}}|f(x-z)| \int_{\mathbf{R}}|\varphi(t)||t|^{-n}\left(1-\left|\frac{z}{t}\right|^{2}\right)_{+}^{\varepsilon-1} d t d z .
$$

If we can show that the kernel

$$
\int_{\mathbf{R}}|\varphi(t)||t|^{-n}\left(1-\left|\frac{z}{t}\right|^{2}\right)_{+}^{\varepsilon-1} d t
$$

of the convolution of the right-hand side is bounded, then

$$
\sup _{x}\left\|\varphi F_{x}^{\varepsilon-1+i \mu}\right\|_{1} \leq C_{\varepsilon} e^{3 \pi|\mu|}\|f\|_{1} .
$$

But by the trivial estimate $\left(1-\left|\frac{z}{t}\right|^{2}\right)_{+}^{\varepsilon-1} \leq\left(1-\left|\frac{z}{t}\right|\right)_{+}^{\varepsilon-1}$ and splitting the integral defining the kernel in two parts, we can find a bound of the kernel

$$
\begin{aligned}
& \int_{|t| \geq|z|}|\varphi(t)||t|^{-n}\left(1-\left|\frac{z}{t}\right|^{2}\right)^{\varepsilon-1} d t \leq \int_{|t| \geq|z|}|\varphi(t)||t|^{-n}\left(1-\left|\frac{z}{t}\right|\right)^{\varepsilon-1} d t \\
&=\int_{|t|>2|z|}|\varphi(t)||t|^{-n}\left(1-\left|\frac{z}{t}\right|\right)^{\varepsilon-1} d t+\int_{|z| \leq|t| \leq 2|z|}|\varphi(t)||t|^{-n}\left(1-\left|\frac{z}{t}\right|\right)^{\varepsilon-1} d t \\
& \leq \int_{|t|>2|z|}|\varphi(t)||t|^{-n} 2^{1-\varepsilon} d t+\int_{|z| \leq|t| \leq 2|z|}|\varphi(t)||t|^{-n+1}|t|^{-1}\left(1-\left|\frac{z}{t}\right|\right)^{\varepsilon-1} d t \\
& \leq 2^{1-\varepsilon} \int_{|t|>2|z|}|\varphi(t)||t|^{-n} d t \\
&+\left(\sup _{|z| \leq|t| \leq 2|z|}|\varphi(t)||t|^{-n+1}\right) \int_{\frac{1}{2} \leq|z / t| \leq 1}\left(1-\left|\frac{z}{t}\right|\right)^{\varepsilon-1}|t|^{-1} d t .
\end{aligned}
$$

Changing variable $s=\frac{|z|}{t}$ in the second integral makes it equal to

$$
\int_{\frac{1}{2} \leq|s| \leq 1}(1-|s|)^{\varepsilon-1}\left|\frac{s}{z}\right| \frac{|z|}{s^{2}} d s \leq 4 \int_{\frac{1}{2}}^{1}(1-s)^{\varepsilon-1} d s=\frac{4 \cdot 2^{\varepsilon}}{\varepsilon} .
$$

Summing up we see that the kernel is bounded if $\varphi \in C_{0}^{\infty}(\mathbf{R} \backslash\{0\})$. We now use an extended version of Stein's interpolation theorem for a complex family of operators (see [BP, p. 313]) to get

$$
\left(\int_{\mathbf{R}^{n}}\left\|\varphi F_{x}^{\gamma}\right\|_{p}^{p^{\prime}} d x\right)^{\frac{1}{p^{\prime}}} \leq C\|f\|_{p}
$$


$p=\frac{n-1+2 \varepsilon}{n+\gamma+\varepsilon}$, from (17) and the earlier used $L^{2}$ estimate

$$
\left(\int_{\mathbf{R}^{n}}\left\|\varphi F_{x}^{-\frac{n+1}{2}+i \mu}\right\|_{2}^{2} d x\right)^{\frac{1}{2}} \leq C e^{3 \pi|\mu|}\|\varphi\|_{2}\|f\|_{2}=C e^{3 \pi|\mu|}\|f\|_{2} .
$$

Let $\gamma$ be fixed in $\left[-\frac{n+1}{2},-1\right]$. Using Riesz-Thorin's theorem for vector-valued functions (see [BL, p. 107]) we can interpolate between

$$
\left(\int_{\mathbf{R}^{n}}\left\|\varphi F_{x}^{\gamma}\right\|_{p}^{p} d x\right)^{\frac{1}{p}} \leq C\|\varphi\|_{p}\|f\|_{p}
$$

where $p=\frac{n-1+2 \varepsilon}{n+\gamma+\varepsilon}>1$ and (1) for $\alpha=\beta=\frac{n+1}{2}+\gamma$, i.e.

$$
\left(\int_{\mathbf{R}^{n}}\left\|\varphi F_{x}^{\gamma}\right\|_{\mathscr{L}_{\beta}^{2}}^{2} d x\right)^{\frac{1}{2}} \leq C\|\varphi\|_{\tilde{\mathscr{L}}_{\beta}^{2}}\|f\|_{2}
$$

and obtain

$$
\left(\int_{\mathbf{R}^{n}}\left\|\varphi F_{x}^{\gamma}\right\|_{\mathscr{L}_{\alpha}^{p}}^{p} d x\right)^{\frac{1}{p}} \leq C\|\varphi\|_{p_{0}}^{1-\theta}\|\varphi\|_{\tilde{\mathscr{L}}_{\beta}^{2}}^{\theta}\|f\|_{p}
$$

Here $\frac{n-1+2 \varepsilon}{n+\gamma+\varepsilon}=p_{0} \leq p \leq 2, \alpha=\frac{n-1}{p^{\prime}}+\gamma+1+\varepsilon\left(1-\frac{2}{p}\right)$ and $\theta=\frac{\alpha}{\beta}$.

Using the same argument we can interpolate between (20) with $p$ such that $p^{\prime}=\frac{p}{p-1}=\frac{n-1+2 \varepsilon}{n+\gamma+\varepsilon}<\infty$ and (21) to obtain (22) with $2 \leq p \leq p_{0}^{\prime}=-\frac{n-1+2 \varepsilon}{1+\gamma-\varepsilon}$, $\alpha=\frac{n-1}{p}+\gamma+1+\varepsilon\left(1-\frac{2}{p^{\prime}}\right)$ and $\theta=\frac{\alpha}{\beta}$.

We now continue with the interpolation of (18), $p=\frac{n-1+2 \varepsilon}{n+\gamma+\varepsilon}$, and (21). This yields

$$
\left(\int_{\mathbf{R}^{n}}\left\|\varphi F_{x}^{\gamma}\right\|_{\mathscr{L}_{\alpha}^{p}}^{p^{\prime}} d x\right)^{\frac{1}{p^{\prime}}} \leq C_{\varphi}\|f\|_{p}
$$

for $\frac{n-1+2 \varepsilon}{n+\gamma+\varepsilon} \leq p \leq 2$ and $\alpha=\frac{n-1}{p^{\prime}}+\gamma+1+\varepsilon\left(1-\frac{2}{p}\right)$. Another application of the above type of Riesz-Thorin's interpolation theorem, now applied to (22) and (23), gives

$$
\left(\int_{\mathbf{R}^{n}}\left\|\varphi F_{x}^{\gamma}\right\|_{\mathscr{L}_{\alpha}^{p}}^{r} d x\right)^{\frac{1}{r}} \leq C_{\varphi}\|f\|_{p}
$$

Here $\frac{n-1+2 \varepsilon}{n+\gamma+\varepsilon} \leq p \leq 2, p \leq r \leq p^{\prime}$ and $\alpha=\frac{n-1}{p^{\prime}}+\gamma+1+\varepsilon\left(1-\frac{2}{p}\right)$ ( $\gamma$ fixed). But since the spaces $\mathscr{L}_{\alpha}^{p}(\mathbf{R})$ decrease when $\alpha$ increases, the conclusion still holds if we allow $0 \leq \alpha \leq \frac{n-1}{p^{\prime}}+\gamma+1+\varepsilon\left(1-\frac{2}{p}\right)$ (or $0 \leq \alpha \leq \frac{n-1}{p}+\gamma+1+\varepsilon\left(1-\frac{2}{p^{\prime}}\right)$ if $p \geq 2$ ). So, for an arbitrary $p$ such that $\frac{n-1}{n+\gamma}<p \leq 2$ and $0 \leq \alpha<\frac{n-1}{p^{\prime}}+\gamma+1$ we choose a small positive $\varepsilon$ so that $\frac{n-1}{n+\gamma}<\frac{n-1+2 \varepsilon}{n+\gamma+\varepsilon} \leq p \leq 2$ and $\alpha \leq \frac{n-1}{p^{\prime}}+\gamma+1+\varepsilon\left(1-\frac{2}{p}\right)<$ $\frac{n-1}{p^{\prime}}+\gamma+1$. The same thing is done for $2 \leq p<-\frac{n-1}{1+\gamma}$ and $0 \leq \alpha<\frac{n-1}{p}+\gamma+1$.

This proves (5) under the conditions (viii), (ix) and (x) (or (viii), (ix') and $\left(\mathrm{x}^{\prime}\right)$ ).

We now prove (5) under the assumption that $\alpha \leq \frac{n-1}{p^{\prime}}+\gamma+1$ and the restriction $r=p$ and $\gamma$ an integer.

Let $M(A, B)$ be the class of multipliers that give bounded operators from $A$ to $B$, and set $M_{p}=M\left(L^{p}, L^{p}\right)$. The estimate $\|f * d S\|_{1} \leq C\|f\|_{1}$ is easy, since 
the convolution with a finite measure is bounded in $L^{1}\left(\mathbf{R}^{n}\right)$. This shows that the corresponding multiplier

$$
m(|\xi|)=\widehat{(d S})(\xi)=C|\xi|^{-\frac{n}{2}+1} J_{\frac{n}{2}-1}(|\xi|) \in M_{1} .
$$

Now a computation (see [Pr and $\mathbf{E}$, Volume 2, p. 11]) of the derivative of the multiplier gives

$$
\frac{d m}{d r}(r)=-r^{-\frac{n}{2}+1} J_{\frac{n}{2}}(r)=\left(\sum_{i=1}^{n} R_{i}\left(x_{i} d S(x)\right)\right)^{\hat{}}(r),
$$

where $r=|\xi|$ and $R_{i}$ are the Riesz transforms, defined by $\left(\widehat{R_{i} f}\right)(\xi)=\frac{\xi_{i}}{|\xi|} \hat{f}(\xi)$, $i=1, \ldots, n$. So the new operator looks as follows:

$$
\left(\sum_{i=1}^{n} R_{i}\left(x_{i} d S(x)\right)\right) * f=\sum_{i=1}^{n}\left(\left(R_{i}\left(x_{i} d S(x)\right) * f\right)=\sum_{i=1}^{n}\left(\left(R_{i} f\right) *\left(x_{i} d S(x)\right) .\right.\right.
$$

The convolution of a function with the measure $x_{i} d S(x)$ is bounded on $L^{1}\left(\mathbf{R}^{n}\right)$, since it is finite. The Riesz transforms are bounded on $H^{1}\left(\mathbf{R}^{n}\right)$ (see [St1, p. 232]). Thus the operator $\sum_{i=1}^{n}\left(\left(R_{i} f\right) *\left(x_{i} d S(x)\right)\right.$ from $H^{1}\left(\mathbf{R}^{n}\right)$ to $L^{1}\left(\mathbf{R}^{n}\right)$ is bounded, or equivalently $|\xi|^{-\frac{n}{2}+1} J_{\frac{n}{2}}(|\xi|) \in M\left(H^{1}, L^{1}\right)$. If

Lemma 2. Assume that $-\frac{n+1}{2} \leq \gamma \leq-1, p(\gamma)=\frac{n-1}{n+\gamma}$ and $0<\lambda \leq \frac{n+1}{2}+\gamma$.

$$
|\xi|^{-\frac{n}{2}-\gamma} J_{\frac{n}{2}+\gamma}(|\xi|) \in M_{p(\gamma)}
$$

then

$$
|\xi|^{\lambda}|\xi|^{-\frac{n}{2}-\gamma} J_{\frac{n}{2}+\gamma}(|\xi|) \in M_{p(\gamma-\lambda)} .
$$

If

then

$$
|\xi|^{-\frac{n}{2}-\gamma} J_{\frac{n}{2}+\gamma+1}(|\xi|) \in M_{p(\gamma)}, \quad \gamma<-1,
$$

$$
|\xi|^{\lambda}|\xi|^{-\frac{n}{2}-\gamma} J_{\frac{n}{2}+\gamma+1}(|\xi|) \in M_{p(\gamma-\lambda)} .
$$

Assume for a moment the truth of this lemma. We use induction to prove our assertion. The induction hypothesis is

$$
r^{-\frac{n}{2}+1+k} J_{\frac{n}{2}-1-k}(r), r^{-\frac{n}{2}+1+k} J_{\frac{n}{2}-k}(r) \in M_{p(-k-1)},
$$

where $k=1,2, \ldots, m-1$ and $m \leq \frac{n+1}{2}$. We shall show that (24) holds true even for $k=m$.

With use of the recursion formula $J_{\nu-1}(r)=\frac{2 \nu}{r} J_{\nu}(r)-J_{\nu+1}(r)$ (see [E, Volume 2, p. 12]) we obtain

$$
\begin{aligned}
& r^{-\frac{n}{2}+1+m} J_{\frac{n}{2}-1-m}(r) \\
& \quad=r^{-\frac{n}{2}+1+m}\left[\frac{2\left(\frac{n}{2}+m\right)}{r} J_{\frac{n}{2}-1-(m-1)}(r)-J_{\frac{n}{2}-1-(m-2)}(r)\right] \\
& \quad=(n+2 m) r^{-\frac{n}{2}+1+(m-1)} J_{\frac{n}{2}-1-(m-1)}(r)-r \cdot r^{-\frac{n}{2}+1+(m-1)} J_{\frac{n}{2}-1-(m-2)}(r)
\end{aligned}
$$

The first term belongs to $M_{p(-m)}$ according to the assumption, but $M_{p(-m)} \subset$ $M_{p(-m-1)}$ by duality and interpolation. See [BL, p. 133]. The second term 
belongs to $M_{p(-m-1)}$, because

$$
r^{-\frac{n}{2}+1+(m-1)} J_{\frac{n}{2}-1-(m-2)}(r) \in M_{p(-m)}
$$

by the assumption and this together with the lemma gives

$$
r \cdot r^{-\frac{n}{2}+1+(m-1)} J_{\frac{n}{2}-1-(m-2)}(r) \in M_{p(-m-1)} .
$$

Therefore, $r^{-\frac{n}{2}+1+m} J_{\frac{n}{2}-1-m}(r) \in M_{p(-m-1)}$.

Consider now

$$
r^{-\frac{n}{2}+1+m} J_{\frac{n}{2}-m}(r)=r \cdot r^{-\frac{n}{2}+1+(m-1)} J_{\frac{n}{2}-1-(m-1)}(r) .
$$

But according to the assumption

$$
r^{-\frac{n}{2}+1+(m-1)} J_{\frac{n}{2}-1-(m-1)}(r) \in M_{p(-m)}
$$

and a multiplication with $r$ puts the multiplier in the class $M_{p(-m-1)}$ by the lemma. Thus (24) is true for $k=m$. This proves the induction step. To conclude the starting point $k=1$, we observe that it follows from the fact that

$$
r^{-\frac{n}{2}+1} J_{\frac{n}{2}-1}(r) \in M_{1} \quad \text { and } \quad r^{-\frac{n}{2}+1} J_{\frac{n}{2}}(r) \in M\left(H^{1}, L^{1}\right) .
$$

Applying the above argument and the inclusions $M_{p(-1)}=M_{1} \subset M\left(H^{1}, L^{1}\right) \subset$ $M_{p(-2)}$. (25) can be interpreted as step $k=0$. But the assumption (24) implies that $\left\|F^{\gamma}(1)\right\|_{p} \leq C\|f\|_{p}$, for integral $\gamma$. By the same homogeneity argument as before one easily sees that

$$
\left\|F^{\gamma}(t)\right\|_{p} \leq C\|f\|_{p}, p=p(\gamma),
$$

with $C$ independent of $t$, and as a consequence, for $r=p$,

$$
\left(\int_{\mathbf{R}^{n}}\left\|\varphi F_{x}^{\gamma}\right\|_{p}^{r} d x\right)^{\frac{1}{r}}=\left(\int_{\mathbf{R}}|\varphi(t)|^{p}\left\|F^{\gamma}(t)\right\|_{p}^{p} d t\right)^{\frac{1}{p}} \leq C\|\varphi\|_{p}\|f\|_{p} .
$$

Thus (5) follows from the above mentioned interpolation with (1) $(\alpha=\beta)$.

We next assume that $\alpha \leq \frac{n-1}{p^{\prime}}+\gamma+1, r=p$ and $\frac{n+1}{2}+\gamma$ is equal to an integer. For such $\gamma$ 's $J_{\frac{n}{2}+\gamma}$ becomes a "spherical" Bessel function and the remainder term in its asymptotic expansion vanishes (see [E, Volume 2, p. 78]), i.e.

$$
J_{\frac{n}{2}+\gamma}(|\xi|)=\sum_{i=0}^{k}\left(c_{i} e^{i|\xi|}+d_{i} e^{-i|\xi|}\right)|\xi|^{-\frac{1}{2}-i} .
$$

Let $\phi$ be a cut-off function in $C_{0}^{\infty}(\mathbf{R})$ such that $\phi(|\xi|)=1$ for $|\xi|<1$ and $\phi(|\xi|)=0$ for $|\xi|>2$. Then

$$
\phi(|\xi|)|\xi|^{-\frac{n}{2}-\gamma} J_{\frac{n}{2}+\gamma}(|\xi|) \in M_{p}, p \geq 1,
$$

since $|\xi|^{-\frac{n}{2}-\gamma} J_{\frac{n}{2}+\gamma}(|\xi|)$ is $C^{\infty}$ and bounded for small $\xi$ if $\gamma \geq-\frac{n+1}{2}$. It will be enough to find an estimate for the first term $(1-\phi(|\xi|))\left(c_{0} e^{i|\xi|}+d_{0} e^{-i|\xi|}\right)|\xi|^{-\frac{n+1}{2}-\gamma}$ in

$$
\begin{aligned}
&(1-\phi(|\xi|))|\xi|^{-\frac{n}{2}-\gamma} J_{\frac{n}{2}+\gamma}(|\xi|) \\
&=(1-\phi(|\xi|))\left(c_{0} e^{i|\xi|}+d_{0} e^{-i|\xi|}\right)|\xi|^{-\frac{n+1}{2}-\gamma} \\
&+\cdots+(1-\phi(|\xi|))\left(c_{k} e^{i|\xi|}+d_{k} e^{-i|\xi|}\right)|\xi|^{-\frac{n+1}{2}-\gamma-k}
\end{aligned}
$$


since the others decay faster. $k$ is a number depending on $\frac{n}{2}+\gamma$ only. But such an estimate falls under the scope of Theorem 1 in [Mi] and as a consequence we get that the first term belongs to $M\left(H^{p}, H^{p}\right)$ if

$$
(n-1)\left|\frac{1}{p}-\frac{1}{2}\right| \leq \frac{n+1}{2}+\gamma .
$$

This means that $\left\|F^{\gamma}(1)\right\|_{p} \leq C\|f\|_{p}, 1<p<\infty$, if $-\frac{n+1}{2} \leq \gamma \leq-1$ and $\frac{n-1}{n+\gamma} \leq$ $p \leq 2$ (or $\frac{n-1}{n+\gamma} \leq p^{\prime} \leq 2$ ). $\left\|F^{-1}(1)\right\|_{1} \leq C\|f\|_{1}$ is contained in the above case where $\gamma$ is an integer. From this we obtain, as before, (5) under the desired conditions by interpolation with $(1)(\alpha=\beta)$.

PROOF OF LEMMA 2. It is known that $|\xi|^{i \mu} \in M\left(H^{1}, H^{1}\right) \subset M\left(H^{1}, L^{1}\right)$, $\mu \in \mathbf{R}$, with

$$
\left\||\xi|^{i \mu}\right\|_{M\left(H^{1}, L^{1}\right)} \leq C(1+|\mu|)^{n+1} .
$$

$\left(|\xi|^{i \mu}\right.$ satisfies Hörmander's hypothesis for the Mihlin multiplier theorem and gives rise to an operator bounded on $H^{1}\left(\mathbf{R}^{n}\right)$. See [FS, p. 159].) This implies that

$$
|\xi|^{i \mu-\frac{n}{2}+1} J_{\frac{n}{2}-1}(|\xi|) \in M\left(H^{1}, L^{1}\right)
$$

because

$$
|\xi|^{-\frac{n}{2}+1} J_{\frac{n}{2}-1}(|\xi|)=C \widehat{(d S)}(\xi) \in M_{1} .
$$

We have that $M\left(H^{1}, L^{1}\right) \subset M_{p(\gamma)}$ if $-\frac{n+1}{2} \leq \gamma \leq-1$, and as a consequence

$$
|\xi|^{i \mu-\frac{n}{2}-\gamma} J_{\frac{n}{2}+\gamma}(|\xi|) \in M_{p(\gamma)}
$$

if

$$
|\xi|^{-\frac{n}{2}-\gamma} J_{\frac{n}{2}+\gamma}(|\xi|) \in M_{p(\gamma)} .
$$

In the other endpoint we have that

$$
\left.|| \xi\right|^{\frac{n+1}{2}+\gamma+i \mu}|\xi|^{-\frac{n}{2}-\gamma} J_{\frac{n}{2}+\gamma}(|\xi|)|=||\xi|^{\frac{1}{2}} J_{\frac{n}{2}+\gamma}(|\xi|) \mid \leq C,
$$

if $\gamma \geq-\frac{n+1}{2}$. Cf. Lemma $1(l=0)$. Thus

$$
|\xi|^{\frac{n+1}{2}+\gamma+i \mu}|\xi|^{-\frac{n}{2}-\gamma} J_{\frac{n}{2}+\gamma}(|\xi|) \in M_{2},
$$

with the $M_{2}$-norm independent of $\mu$. Interpolating the complex family of operators, defined by the multipliers $|\xi|^{\lambda+i \mu}|\xi|^{-\frac{n}{2}-\gamma} J_{\frac{n}{2}+\gamma}(|\xi|)$, between the endpoints $\lambda=0$ and $\lambda=\frac{n+1}{2}+\gamma$ gives

$$
|\xi|^{\lambda}|\xi|^{-\frac{n}{2}-\gamma} J_{\frac{n}{2}+\gamma}(|\xi|) \in M_{p(\gamma-\lambda)}
$$

$0<\lambda \leq \frac{n+1}{2}+\gamma$. If

$$
|\xi|^{-\frac{n}{2}-\gamma} J_{\frac{n}{2}+\gamma+1}(|\xi|) \in M_{p(\gamma)}, \quad \gamma<-1, \quad \text { or } \quad|\xi|^{-\frac{n}{2}+1} J_{\frac{n}{2}}(|\xi|) \in M\left(H^{1}, L^{1}\right)
$$

we replace $J_{\frac{n}{2}+\gamma}\left(J_{\frac{n}{2}-1}\right)$ by $J_{\frac{n}{2}+\gamma+1}\left(J_{\frac{n}{2}}\right)$ in the previous discussion and obtain

$$
|\xi|^{\lambda}|\xi|^{-\frac{n}{2}-\gamma} J_{\frac{n}{2}+\gamma+1}(|\xi|) \in M_{p(\gamma-\lambda)}
$$

for $0<\lambda \leq \frac{n+1}{2}+\gamma$.

PROOF OF COROLlaRY 2. As in the proof of Corollary 1 we use that $\mathscr{L}_{\alpha}^{p}$ is continuously embedded in $L^{q}, B M O$ and $\Lambda_{\delta}$ for certain values of $p, \alpha, q$ and $\delta$. 
For $\frac{1}{q}=\frac{1}{p}-\alpha$ and $1<p \leq q<\infty$ we have the embedding $\mathscr{L}_{\alpha}^{p}(\mathbf{R}) \subset L^{q}(\mathbf{R})$. See [BL, p. 153]. So for $\gamma, p$ and $\alpha$ satisfying (vii), (ix) and (x) this becomes true if

$$
\frac{1}{q}=\frac{1}{p}-\alpha>\frac{1}{p}-\frac{n-1}{p^{\prime}}-\gamma-1=-\left(\frac{n}{p^{\prime}}+\gamma\right) \geq 0,
$$

which is (xi). If $p$ and $\alpha$ satisfy (ix') and ( $\left.\mathrm{x}^{\prime}\right)$ instead of (ix) and (x), $q$ is then forced to satisfy

$$
\frac{1}{q}=\frac{1}{p}-\alpha>\frac{1}{p}-\frac{n-1}{p}-\gamma-1=-\left(\frac{n-2}{p}+\gamma+1\right) \geq 0 .
$$

This is $\left(\mathrm{xi}^{\prime}\right)$ and proves that (xi) or (xi') is sufficient for (6).

If $\alpha=\frac{1}{p}$ and $1<p<\infty$, the space $\mathscr{L}_{\alpha}^{p}(\mathbf{R})$ embeds continuously in $B M O(\mathbf{R})$. See [St1, p. 164]. This substitutes the endpoint $q=\infty$ in the previous case, and by the same reasons $(7)$ is true if

$$
-\left(\frac{n}{p^{\prime}}+\gamma\right) \geq 0 \quad(p \leq 2) \quad \text { or } \quad-\left(\frac{n-2}{p}+\gamma+1\right) \geq 0 \quad(p \geq 2)
$$

which is contained in (xii) and (xii').

In the proof of (8) we need the following embedding $\mathscr{L}_{\alpha}^{p}(\mathbf{R}) \subset \Lambda_{\delta}(\mathbf{R}), \alpha=\frac{1}{p}+\delta$, $1<p<\infty, \delta>0$, which can be obtained from the chain of embeddings

$$
\mathscr{L}_{\frac{1}{p}+\delta}^{p} \subset \Lambda_{\frac{1}{p}+\delta}^{p 2} \subset \Lambda_{\frac{1}{p}+\delta}^{p \infty} \subset \Lambda_{\delta}^{\infty \infty}=\Lambda_{\delta}, \quad p \leq 2,
$$

or

$$
\mathscr{L}_{\frac{1}{p}+\delta}^{p} \subset \Lambda_{\frac{1}{p}+\delta}^{p p} \subset \Lambda_{\frac{1}{p}+\delta}^{p \infty} \subset \Lambda_{\delta}^{\infty \infty}=\Lambda_{\delta}, p \geq 2 .
$$

(The definition of the Lipschitz-Besov spaces $\Lambda_{s}^{p q}$ and the embeddings can be found in [St1, Chapter V, §5-6].) With $\alpha$ satisfying (x) we have

$$
\frac{1}{p}+\delta=\alpha<\frac{n-1}{p^{\prime}}+\gamma+1
$$

and therefore $\delta<\frac{n}{p^{\prime}}+\gamma$ so that if $\frac{n}{n+\gamma}<p \leq 2$ the conditions for the embedding are satisfied. This proves (8) in the case (xiii). For $\alpha$ satisfying $\left(\mathrm{x}^{\prime}\right)$ we get that

$$
\delta<\frac{n-2}{p}+\gamma+1
$$

and (5) if

$$
2 \leq p<-\frac{n-2}{1+\gamma}
$$

PROOF OF COROLLARY 3 . We recall the estimates in the proof of Theorem 2 and take a closer look at the dependence of $\varphi$.

Another estimate of the kernel

$$
\int_{\mathbf{R}}|\varphi(t)||t|^{-n}\left(1-\left|\frac{z}{t}\right|^{2}\right)_{+}^{\varepsilon-1} d t
$$

gives

$$
C\left(\left\|\varphi|\cdot|^{-n}\right\|_{1}+\left\|\varphi|\cdot|^{-n+1}\right\|_{\infty}\right)
$$


as an upper bound. Therefore (17) can be rewritten to yield

$$
\sup _{x}\left\|\varphi F_{x}^{\varepsilon-1+i \mu}\right\|_{1} \leq C_{\varepsilon} e^{3 \pi|\mu|} C\left(\left\|\varphi|\cdot|^{-n}\right\|_{1}+\left\|\varphi|\cdot|^{-n+1}\right\|_{\infty}\right)\|f\|_{1} .
$$

Interpolating with the $L^{2}$ estimate (19) of the endpoint $\gamma=-\frac{n+1}{2}+i \mu$ gives

$$
\left(\int_{\mathbf{R}^{n}}\left\|\varphi F_{x}^{\gamma}\right\|_{p}^{p^{\prime}} d x\right)^{\frac{1}{p^{\prime}}} \leq C\left(\left\|\varphi|\cdot|^{-n}\right\|_{1}+\left\|\varphi|\cdot|^{-n+1}\right\|_{\infty}\right)^{1-\theta_{1}}\|\varphi\|_{2}^{\theta_{1}}\|f\|_{p}
$$

where

$$
p=\frac{n-1+2 \varepsilon}{n+\gamma+\varepsilon}
$$

and

$$
\theta_{1}=\frac{2}{p^{\prime}}=-2 \frac{1+\gamma+\varepsilon}{n-1+2 \varepsilon} \text {. }
$$

We continue, as in the proof of Theorem 2 , by the interpolation between (26) and (21) with the following result (replacing (23)):

$$
\begin{aligned}
& \left(\int_{\mathbf{R}^{n}}\left\|\varphi F_{x}^{\gamma}\right\|_{\mathscr{L}_{\alpha}^{p}}^{p^{\prime}} d x\right)^{\frac{1}{p^{\prime}}} \\
& \quad \leq C\left(\left(\left\|\varphi|\cdot|^{-n}\right\|_{1}+\left\|\varphi|\cdot|^{-n+1}\right\|_{\infty}\right)^{1-\theta_{1}}\|\varphi\|_{2}^{\theta_{1}}\right)^{1-\theta_{2}}\|\varphi\|_{\tilde{\mathscr{L}}_{\beta}^{2}}^{\theta_{2}}\|f\|_{p} .
\end{aligned}
$$

Here $\frac{n-1+2 \varepsilon}{n+\gamma+\varepsilon}=p_{0} \leq p \leq 2, \alpha=\frac{n-1}{p^{\prime}}+\gamma+1+\varepsilon\left(1-\frac{2}{p}\right), \beta=\frac{n+1}{2}+\gamma$ and $\theta_{2}=\frac{\alpha}{\beta}$. Finally, we interpolate (27) with (22) $\left(\theta=\theta_{2}\right)$ in the case when $p \leq 2$ and obtain

$$
\begin{aligned}
\left(\int_{\mathbf{R}^{n}}\right. & \left.\left\|\varphi F_{x}^{\gamma}\right\|_{\mathscr{L}_{\alpha}^{p}}^{r} d x\right)^{\frac{1}{r}} \\
\leq & C\left(\left(\left(\left\|\varphi|\cdot|^{-n}\right\|_{1}+\left\|\varphi|\cdot|^{-n+1}\right\|_{\infty}\right)^{1-\theta_{1}}\|\varphi\|_{2}^{\theta_{1}}\right)^{1-\theta_{2}}\|\varphi\|_{\tilde{\mathscr{L}}_{\beta}^{2}}^{\theta_{2}}\right)^{1-\theta_{3}} \\
& \times\left(\|\varphi\|_{p_{0}}^{1-\theta_{2}}\|\varphi\|_{\check{\mathscr{L}}_{\beta}^{2}}^{\theta_{2}}\right)^{\theta_{3}}\|f\|_{p} .
\end{aligned}
$$

Where $p \leq r \leq p^{\prime}$ and

$$
\theta_{3}=\frac{\frac{1}{r}+\frac{1}{p}-1}{\frac{2}{p}-1}
$$

Choose $\rho_{0} \in C_{0}^{\infty}(\mathbf{R} \backslash\{0\})$ so that supp $\rho_{0} \subset\left\{t ; \frac{1}{2}<|t|<2\right\}$ and

$$
\sum_{k=-\infty}^{\infty} \rho_{0}\left(2^{k} t\right)=1, \quad t \neq 0
$$

Set $\rho_{k}(t)=\rho_{0}\left(2^{k} t\right), k \in \mathbf{Z}$, and

$$
\rho(t)=\sum_{k=0}^{\infty} \rho_{0}\left(2^{k} t\right),
$$

then $\rho(t)=1$ for $0<|t| \leq 1$. It is sufficient to prove that (5) holds with $\rho(t)|t|^{\eta}$ instead of $\varphi(t)|t|^{\eta}$. We first obtain (5) with $\rho_{k}(t)|t|^{\eta}$ and then the full result by summing them up. Here is where the above estimates come in. Replacing $\varphi$ by 
$\rho_{k}|\cdot|^{\eta}$ in (28) gives that

$$
\left(\left(\left(\left\|\varphi|\cdot|^{-n}\right\|_{1}+\left\|\varphi|\cdot|^{-n+1}\right\|_{\infty}\right)^{1-\theta_{1}}\|\varphi\|_{2}^{\theta_{1}}\right)^{1-\theta_{2}}\|\varphi\|_{\mathscr{\mathscr { L }}_{\beta}^{2}}^{\theta_{2}}\right)^{1-\theta_{3}}\left(\|\varphi\|_{p_{0}}^{1-\theta_{2}}\|\varphi\|_{\tilde{\mathscr{L}}_{\beta}^{2}}^{\theta_{2}}\right)^{\theta_{3}}
$$

can be estimated and an upper bound is a constant times a power of $2^{-k}$. If $\eta$ is chosen so that the exponent becomes positive, then the geometric series converges and we obtain (5).

Therefore, we proceed with the estimates of the norms of $\rho_{k}|\cdot|^{\eta}$

$$
\begin{gathered}
\left\|\rho_{k}|\cdot|^{\eta}|\cdot|^{-n}\right\|_{1}+\left\|\rho_{k}|\cdot|^{\eta}|\cdot|^{-n+1}\right\|_{\infty}=C\left(2^{-k}\right)^{\eta-n+1}, \\
\left\|\rho_{k}|\cdot|^{\eta}\right\|_{p_{0}}=C\left(2^{-k}\right)^{\eta+\frac{1}{p_{0}}}
\end{gathered}
$$

and

$$
\left\|\rho_{k}|\cdot|^{\eta}\right\|_{\tilde{\mathscr{E}}_{\beta}^{2}} \leq C\left(2^{-k}\right)^{\eta-\beta+\frac{1}{2}}
$$

So the considered exponent becomes

$$
\begin{aligned}
{[[(\eta-n+1)} & \left.\left.\left(1-\theta_{1}\right)+\left(\eta+\frac{1}{2}\right) \theta_{1}\right]\left(1-\theta_{2}\right)+\left(\eta-\beta+\frac{1}{2}\right) \theta_{2}\right]\left(1-\theta_{3}\right) \\
& +\left[\left(\eta+\frac{1}{p_{0}}\right)\left(1-\theta_{2}\right)+\left(\eta-\beta+\frac{1}{2}\right) \theta_{2}\right] \theta_{3} \\
= & \eta+\left[\left[(1-n)\left(1-\theta_{1}\right)+\frac{\theta_{1}}{2}\right]\left(1-\theta_{2}\right)+\left(\frac{1}{2}-\beta\right) \theta_{2}\right]\left(1-\theta_{3}\right) \\
& +\left[\frac{1}{p_{0}}\left(1-\theta_{2}\right)+\left(\frac{1}{2}-\beta\right) \theta_{2}\right] \theta_{3} \\
= & \eta+\left[(1-n)\left(1-\theta_{1}\right)+\frac{\theta_{1}}{2}\right]\left(1-\theta_{2}\right)\left(1-\theta_{3}\right) \\
& +\frac{1}{p_{0}}\left(1-\theta_{2}\right) \theta_{3}+\left(\frac{1}{2}-\beta\right) \theta_{2} .
\end{aligned}
$$

Since we only consider positive exponents we can take $\varepsilon=0$ where it appears above.

Performing the substitutions

$$
\begin{gathered}
p_{0}=\frac{n-1}{n+\gamma}, \quad \beta=\frac{n+1}{2}+\gamma, \quad \theta_{1}=-2 \frac{1+\gamma}{n-1}, \\
\theta_{2}=\frac{\frac{n-1}{p^{\prime}}+\gamma+1}{\frac{n+1}{2}+\gamma} \quad \text { and } \quad \theta_{3}=\frac{\frac{1}{r}+\frac{1}{p}-1}{\frac{2}{p}-1}
\end{gathered}
$$


in the exponent gives

$$
\begin{aligned}
& \eta+\left((1-n)\left(1+2 \frac{1+\gamma}{n-1}\right)-\frac{1+\gamma}{n-1}\right)\left(1-\frac{\frac{n-1}{p^{\prime}}+\gamma+1}{\frac{n+1}{2}+\gamma}\right)\left(1-\frac{\frac{1}{r}+\frac{1}{p}-1}{\frac{2}{p}-1}\right) \\
& +\frac{n+\gamma}{n-1}\left(1-\frac{\frac{n-1}{p^{\prime}}+\gamma+1}{\frac{n+1}{2}+\gamma}\right) \frac{\frac{1}{r}+\frac{1}{p}-1}{\frac{2}{p}-1}-\left(\frac{n}{2}+\gamma\right) \frac{\frac{n-1}{p^{\prime}}+\gamma+1}{\frac{n+1}{2}+\gamma} \\
& =\eta+\left(1-n-2-2 \gamma-\frac{1+\gamma}{n-1}\right) \frac{\frac{n+1}{2}+\gamma-\frac{n-1}{p^{\prime}}-\gamma-1}{\frac{n+1}{2}+\gamma} \cdot \frac{\frac{2}{p}-1-\frac{1}{r}-\frac{1}{p}+1}{2\left(\frac{1}{p}-\frac{1}{2}\right)} \\
& +\frac{n+\gamma}{n-1} \cdot \frac{\frac{n+1}{2}+\gamma-\frac{n-1}{p^{\prime}}-\gamma-1}{\frac{n+1}{2}+\gamma} \cdot \frac{\frac{1}{r}+\frac{1}{p}-1}{2\left(\frac{1}{p}-\frac{1}{2}\right)}-\left(\frac{n}{2}+\gamma\right) \frac{\frac{n-1}{p^{\prime}}+\gamma+1}{\frac{n+1}{2}+\gamma} \\
& =\eta+\left(-1-n-2 \gamma-\frac{1+\gamma}{n-1}\right) \frac{(n-1)\left(\frac{1}{p}-\frac{1}{2}\right)}{\frac{n+1}{2}+\gamma} \cdot \frac{\left(\frac{1}{p}-\frac{1}{r}\right)}{2\left(\frac{1}{p}-\frac{1}{2}\right)} \\
& +\frac{n+\gamma}{n-1} \frac{(n-1)\left(\frac{1}{p}-\frac{1}{2}\right)}{\frac{n+1}{2}+\gamma} \cdot \frac{\frac{1}{r}+\frac{1}{p}-1}{2\left(\frac{1}{p}-\frac{1}{2}\right)}-\frac{n-1}{2}(n+2 \gamma) \frac{\frac{1}{p^{\prime}}+\frac{1+\gamma}{n-1}}{\frac{n+1}{2}+\gamma} \\
& =\eta+\frac{n-1}{n+2 \gamma+1}\left[\left(-1-n-2 \gamma-\frac{1+\gamma}{n-1}\right)\left(\frac{1}{p}-\frac{1}{r}\right)\right. \\
& \left.+\frac{n+\gamma}{n-1}\left(\frac{1}{r}+\frac{1}{p}-1\right)-(n+2 \gamma)\left(\frac{1}{p^{\prime}}+\frac{1+\gamma}{n-1}\right)\right] \\
& =\eta+\frac{n-1}{n+2 \gamma+1}[\frac{1}{p} \underbrace{\left(-1-n-2 \gamma-\frac{1+\gamma}{n-1}+\frac{n+\gamma}{n-1}+n+2 \gamma\right)}_{=0} \\
& +\frac{1}{r}\left(1+n+2 \gamma+\frac{1+\gamma}{n-1}+\frac{n+\gamma}{n-1}\right)-\frac{n+\gamma}{n-1} \\
& \left.-(n+2 \gamma)\left(1+\frac{1+\gamma}{n-1}\right)\right] \\
& =\eta+\frac{n-1}{n+2 \gamma+1}\left[\frac{n}{r} \cdot \frac{n+2 \gamma+1}{n-1}-(n+2 \gamma+1) \frac{n+\gamma}{n-1}\right] \\
& =\eta+\frac{n}{r}-(n+\gamma)=\eta-\frac{n}{r^{\prime}}-\gamma \text {. }
\end{aligned}
$$

Which is positive if $\eta>\frac{n}{r^{\prime}}+\gamma$. and

For $p \geq 2$ we repeat the argument for $(22)\left(\theta=\theta_{2}\right)$, but now putting $p_{0}=-\frac{n-1}{1+\gamma}$

$$
\theta_{2}=\frac{\frac{n-1}{p}+\gamma+1}{\frac{n+1}{2}+\gamma}
$$

The exponent becomes

$$
\begin{aligned}
\left(\eta+\frac{1}{p_{0}}\right) & \left(1-\theta_{2}\right)+\left(\eta-\beta+\frac{1}{2}\right) \theta_{2} \\
= & \eta+\frac{1}{p_{0}}\left(1-\theta_{2}\right)+\left(\frac{1}{2}-\beta\right) \theta_{2}
\end{aligned}
$$


and with the substitutions

$$
\begin{aligned}
& \eta-\frac{1+\gamma}{n-1}\left(1-\frac{\frac{n-1}{p}+\gamma+1}{\frac{n+1}{2}+\gamma}\right)-\left(\frac{n}{2}+\gamma\right) \frac{\frac{n-1}{p}+\gamma+1}{\frac{n+1}{2}+\gamma} \\
& =\eta+\frac{1}{p} \cdot \underbrace{\frac{1+\gamma-(n-1)\left(\frac{n}{2}+\gamma\right)}{\frac{n+1}{2}+\gamma}}_{=-n+2} \\
& +(\gamma+1) \underbrace{\left(-\frac{1}{n-1}+\frac{1+\gamma}{(n-1)\left(\frac{n+1}{2}+\gamma\right)}-\frac{\frac{n}{2}+\gamma}{\frac{n+1}{2}+\gamma}\right)}_{=-1} \\
& =\eta-\frac{n-2}{p}-\gamma-1 .
\end{aligned}
$$

Taking $\eta>\frac{n-2}{p}+\gamma+1$ gives a positive exponent. This ends the proof of Corollary 3.

\section{REFERENCES}

[BP] A. Benedek and R. Panzone, The spaces $L^{p}$, with mixed norm, Duke Math. J. 28 (1961), 301-324.

[BL] J. Bergh and J. Löfström, Interpolation spaces, Springer-Verlag, Berlin and New York, 1976.

[Bö] L. Börjeson, Estimates for the Bochner-Riesz operator vith negative index, Reports, Dept. of Math., Univ. of Stockholm, No. 5, 1984.

[B1] J. Bourgain, Estimations de certaines fonctions maximales, C. R. Acad. Sci. Paris 301 (1985), 599-502.

[B2] _ Averages in the plane over convex curves and maximal operators, preprint.

[B3] _ On the spherical maximal function in the plane, preprint.

[CJ] M. Cwikel and S. Janson, Interpolation of analytic families of operators, Studia Math. (to appear).

[E] A. Erdélyi (Editor), Higher transcendental functions, Vols. 1, 2, McGraw-Hill, New York, 1955.

[FS] C. Fefferman and E. M. Stein, $H^{p}$ spaces of several variables, Acta Math. 129 (1972), 137-193.

[LM] J. L. Lions and E. Magenes, Non-homogeneous boundary value problems and applications, Vol. 1, Springer-Verlag, Berlin and New York, 1972.

[Ma] B. Marshall, $L^{p}-L^{q}$ multipliers of anisotropic wave equations, Indiana Univ. Math. J. 33 (1984), 435-457.

[Mi] A. Miyachi, On some estimates for the wave equation in $L^{p}$ and $H^{p}$, J. Fac. Sci. Univ. Tokyo 27 (1980), 331-354.

[OS] D. M. Oberlin and E. M. Stein, Mapping properties of the Radon transform, Indiana Univ. Math. J. 31 (1982), 641-650.

[P] J. Peetre, $H_{p}$ spaces, Lecture notes, Lund, 1974.

[Pr] J. C. Peral, $L^{p}$ estimates for the wave equation, J. Funct. Anal. 36 (1980), 114-145.

[PS] J. Peyrière and P. Sjölin, Regularity of spherical means, Ark. Mat. 16 (1978), 117-126.

[Sj1] P. Sjölin, Lipschitz continuity of spherical means, Linear spaces and approximation, Proc. Conf. Oberwolfach, 1977, Ed. by P. L. Butzer and B. Sz.-Nagy, Birkhäuser, 1978, pp. 229-234.

[Sj2] _ Regularity and integrability of spherical means, Monatsh. Math. 96 (1983), 277-291.

$[\mathbf{S j 3}] \ldots$, Regularity and integrability properties of spherical means, Proc. of the Nineteenth Nordic Congress of Mathematicians, Reykjavik, 1984, pp. 215-218.

[Sj4] _ Regularity properties of spherical means, Proc. A. Haar Memorial Conference (Budapest, 1985) (to appear).

[Sj5] _ _ Norm inequalities for spherical means, Monatsh. Math. 100 (1985), 153-161.

[S8] S. Sjöstrand, On the Riesz means of solutions of Schrödinger equation, Ann. Scuola Norm. Sup. Pisa 24 (1970), 331-348. 
[St1] E. M. Stein, Singular integrals and differentiability properties of functions, Princeton Univ. Press, Princeton, N. J., 1970.

[St2] _ Maximal functions: Spherical means, Proc. Nat. Acad. Sci. U.S.A. 73 (1976), 21742175.

[STW] E. M. Stein, M. H. Taibleson and G. Weiss, Weak type estimates for maximal operators on certain $H^{p}$ classes, Rend. Mat. Circ. Palermo Suppl. 2 (1981), 81-97.

[SWa] E. M. Stein and S. Wainger, Problems in harmonic analysis related to curvature, Bull. Amer. Math. Soc. 84 (1978), 1239-1295.

[SWe] E. M. Stein and G. Weiss, Introduction to Fourier analysis in Euclidean spaces, Princeton Univ. Press, Princeton, N. J., 1971.

[Str] R. Strichartz, Convolutions with kernels having singularities on a sphere, Trans. Amer. Math. Soc. 148 (1970), 461-471.

[T] H. Triebel, Interpolation theory, function spaces, differential operators, North-Holland, Amsterdam, and New York, 1978.

[W] G. N. Watson, Theory of Bessel functions, Cambridge Univ. Press, Cambridge, 1944.

Department of Mathematics, University of Stockholm, Box 6701, S-113 85 STOCKHOLM, SWEDEN 